\section{Intersections}

Canadian Journal of Music

Revue canadienne de musique
Intersections CANADIAN JOURAL OF MUSIC

\title{
Le médium mythologique du Rock'n roll et la musique contemporaine
}

\section{Pierre Albert Castanet}

Volume 32, numéro 1-2, 2012

URI : https://id.erudit.org/iderudit/1018580ar

DOI : https://doi.org/10.7202/1018580ar

Aller au sommaire du numéro

\section{Éditeur(s)}

Canadian University Music Society / Société de musique des universités canadiennes

ISSN

1911-0146 (imprimé)

1918-512X (numérique)

Découvrir la revue

Citer cet article

Castanet, P. A. (2012). Le médium mythologique du Rock'n roll et la musique contemporaine. Intersections, 32(1-2), 83-116. https://doi.org/10.7202/1018580ar
Résumé de l'article

Devenu depuis plus d'un demi-siècle facteur idéologique de mythologie quotidienne, le Rock a séduit bon nombre de compositeurs contemporains (John Adams, Luciano Berio, Steve Martland, Theo Loevendie...). Explorant cette problématique, la communication de Pierre Albert Castanet se fonde sur l'histoire contextuelle et interactive du " populaire " et du " savant ". S'attachant notamment à l'instrumentarium symbolique des rockers (orgue électrique, guitare électrique, guitare basse, batterie), l'article se penche sur quelques études de cas allant des musiques de Pierre Henry à Philippe Manoury en passant par celles de Cathy Berberian, György Ligeti ou Tristan Murail...
Copyright @ C Canadian University Music Society / Société de musique des universités canadiennes, 2013
Ce document est protégé par la loi sur le droit d'auteur. L'utilisation des services d'Érudit (y compris la reproduction) est assujettie à sa politique d'utilisation que vous pouvez consulter en ligne.

https://apropos.erudit.org/fr/usagers/politique-dutilisation/ 


\title{
LE MÉDIUM MYTHOLOGIQUE DU ROCK'N ROLL ET LA MUSIQUE CONTEMPORAINE
}

\author{
Pierre Albert Castanet
}
«Le rock and roll a toujours été traité comme le médium des enfants fous et mutants.» Lou Reed
«Le mythe est toujours un vol de langage.» Roland Barthes

«Populaire, l'art qui porte ce nom ne l'est pas vraiment auprès des philosophes et des théoriciens de la culture, du moins dans la vie professionnelle. Quand il n'est pas ignoré avec superbe, il se voit reprocher son manque de goût et de finesse, et jeté à la poubelle de l'histoire de l'art ${ }^{1} »$. Alors que d'aucuns comprennent que l'entertainment de masse oscille «entre la culture cultivée dont elle serait une variante misérable, vulgarisée, commercialisée, et la culture au sens «ethnosociologique $^{2}$ », il est intéressant de voir comment la musique dite "savante» se confronte aux fredons du peuple comme aux rengaines des collectivités plus ou moins à la mode. Dans ce cadre, certains prônent l'apologie de la domination ${ }^{3}$ alors que d'autres militent pour une légitimité d'ordre culturel ${ }^{4}$.

Ne voyant aucunement de raison de fusion, Benoit Duteurtre a par exemple été frappé par cet esprit de scission qui préside aux destinées de l'esthétique contemporaine. Selon lui, cette dernière sépare le plaisir en deux ordres contradictoires: «celui du bas plaisir hédoniste, consommation sensuelle grossière, développée par l'industrie (plaisir de supermarchés, de sex-shops, volupté monotone des chansons de variétés, humour lourd) ; et celui du plaisir supérieur, élaboration distillée dans les laboratoires, qui se réfère à un contenu contre toute jouissance immédiate (art compliqué, recherche conceptuelle). Cette division constitue le binôme caractéristique des temps modernes; deux dérives artistiques qui grandissent ensemble en s'éloignant toujours l'un de l'autre: le plaisir pauvre vers la simplification, le plaisir élaboré vers l'élaboration 5 ».

Pourtant, sans vouloir remonter aux répertoires des troubadours, trouvères et autres saltimbanques polyvalents, il est relativement aisé d'analyser

\footnotetext{
Shusterman 1991, p. 137.

Morin 1962, p. 241.

Masci 2011.

Richert 2010.

Duteurtre 2006, p. 238.
} 
comment les expressions populaires ${ }^{6}$ ont pu questionner ou séduire les affables compositeurs de «musique sérieuse». Dans le cadre du vingtième siècle, voyez par exemple la part du Jazz ${ }^{7}$ et du Blues relevée et adaptée par certains créateurs (d'Igor Stravinsky à Mark Anthony Turnage en passant par Maurice Ravel, Germaine Tailleferre, Leonard Bernstein... La Monte Young, Alvin Curran, Colon Nancarrow, Terry Jennings, György Ligeti, Marius Constant, Franco Donatoni, John Adams, Michael Finnissy... Antoine Hervé, Christian Lauba, Takayoshi Yoshioka, Brian Wilshere, Franck Bedrossian, Bruno Mantovani, Yann Robin ${ }^{8}$, Pablo Galaz Salamanca, Nicolay Khrust, Amy Williams...). Dans ce sillage, il est aussi facile de noter l'influence circonstanciée du Rap ${ }^{9}$ sur quelques esprits modernistes (de Hugues Dufourt à Andrea Liberovici, via Alain Louvier ou Daniel Mayar.... ${ }^{10}$. Parmi ces esprits ouverts sur le monde des possibles, John Cage - disciple de Marcel Duchamp ${ }^{11}$ - a même tenu à préciser que «l'art n'a pas de raison utilitaire. Il a à voir avec le changement des habitudes dans la perception acoustique ou visuelle et dans l'intellect ${ }^{12}$ ».

À l'instar du Jazz, le Rock'n roll - qui a la particularité d'être également né en marge de l'industrie culturelle ${ }^{13}$ mais qui est passé très rapidement dans la sphère consumériste capitaliste ${ }^{14}$ - montre "désormais une des formes dominantes de la musique populaire ${ }^{15} »$. Et s'il est omniprésent dans les jingles et les rushs publicitaires des médias, il a également phagocyté les folklores et les comptines les plus insolites (on peut à présent écouter par exemple les grandes chansons napolitaines visitées par l'énergie électrifiée du Rock) ${ }^{16}$. En effet, «le son électrique amplifié et distordu est un des ingrédients, un des emblèmes de la réputation violente, incontrôlable et contestataire de la musique Rock. La guitare électrique en est l'origine ${ }^{17}$ », déduit Claude Chastagner. Ex rockeur (comme Danny Elfman ou Hans Zimmer, devenus auteurs de musiques de film), le compositeur danois Casper Cordes a affirmé haut et fort que le Rock - cinquante ans après sa naissance - est devenu un "genre» à part

6 Cf. Castanet 1999 (rééd. 2007), p. 96-103. D’un point de vue général, prière de consulter l'ouvrage de Grignon et Passeron 1989.

7 Pour mémoire, rappelons-nous que Theodor W. Adorno disait en 1967 que le jazz a « entièrement démodé toute la musique de danse non syncopée en la dégradant "en souvenir" " (cf. Adorno mai 1971, p. 8).

8 Élève de Frédéric Durieux et de Jonathan Harvey, Yann Robin est notamment l'auteur de Crescent Scratches (2011). Ce deuxième quatuor à cordes se rapporte aux techniques de jeu développées par les DJ sur leurs platines vinyles que l'on retrouve associés au hip-hop et aux musiques dites « industrielles».

9 "Ainsi donc, le rap est, paraît-il de l'art. Soit, si vous le dîtes. Il est loisible à chacun de préférer les éructations sexistes et antisémites de Public Enemy à un quatuor de Haydn ", écrit Michel Schneider (Schneider 1993, p. 78).

10 Castanet novembre-décembre 2005, p. 29-31.

$11 C f$. Duchamp 1970, p. 116. Pour plus de renseignements relatifs à ces deux artistes, prière de consulter : Stévance 2009.

12 Cf. Lewallen mai 1991, p. 66.

13 Cf. Morin 1994, p. 311

14 Cf. Bell 1976.

15 Ribac 2004, p. 11.

16 Cf. Marengo, Pergolano, De Felicis 2003.

17 Chastagner 2011, p. 108. 
entière: «Je pourrai faire de la musique selon les règles du rock, mais avec un niveau d'abstraction supérieur. $\mathrm{Si}$ je voulais faire une musique exprimant la colère, si je devais écrire une pièce s'intitulant Le droit à la colère, je prendrais peut-être les instruments de la musique rock pour exprimer la colère ${ }^{18}$ ». Dégagée du phénomène de récupération primaire, la règle du jeu ne figure donc pas forcément le formulaire d'une simple réplique, le codex d'une pure imitation ${ }^{19}$ mais implique au mieux une réaction par sympathie pour une sorte d'exhortation vulnérable et perméable - manière de phénomènes assez proches de l'impulsion mimétique, voire des «stimuli sensibles ${ }^{20}$ » relevés en son temps par Theodor W. Adorno.

\section{De la CUlture du Rock}

Arpentant avec lucidité les chemins de la «culture au pluriel», l'historien et anthropologue Michel de Certeau a noté qu'un homme "cultivé» doit être «conforme au modèle élaboré dans les sociétés stratifiées par une catégorie qui a introduit ses normes là où elle imposait son pouvoir ${ }^{21}$ ». Participant au mythe ${ }^{22}$ plénier de la "culture jeune ${ }^{23}$ " au sein de la société d'abondance (au capital anonyme), le Rock a laissé place à des inconditionnels passionnés autant qu'à de farouches contradicteurs. Ainsi, aux yeux ${ }^{24}$ et aux oreilles de Yann Plougastel, ce type de musique, en tant que nouveau médium véhiculaire de la philosophie quotidienne adolescente, est devenu très vite «une culture, c'est-à-dire plus qu'un genre musical, mais une attitude par rapport à la vie et à toutes les formes d'expression artistique ${ }^{25}$ ». En revanche, pour Gaétan Soucy, ce genre «ne saurait être une culture, au sens humaniste, en ceci précisément qu'une civilisation n'existe que dans la mesure où elle a les outils et l'espace philosophique adéquat pour pouvoir se réfléchir elle-même jusque dans ses fondements. Et le rock, par définition, par essence, exclut cette réflexion, exclut toute réflexion. Le rock'n'roll est imprudence en son fondement même ${ }^{26}$ ».

Il n'empêche que - sincères ou imposteurs, libres ou calculateurs? - les compositeurs David Bedford, Cornelius Cardew, Hans Werner Henze ${ }^{27}$, Frank

18 Cordes 2006, p. 32.

19 « Le mirage critique présentant la musique copier-coller-élément-de-décor ou tout simplement l'antimusique, ne marche plus ", remarque Hans-Christian von Dadelsen (cf. Darbon 2007, p. 246).

20 Adorno 1982, p. 7.

21 de Certeau 1993, p. 167.

22 En ce sens, "si la société de consommation ne produit plus de mythe, c'est qu'elle est à ellemême son propre mythe» (Baudrillard 1970, p. 311).

23 Cf. Sabatier 2011.

24 Voyez par exemple l'installation de George Segal intitulée Rock and Roll Combo (1964) montrant des sculptures d'humains en plâtre accompagnée d'une guitare électrique et d'une batterie (cf. Electric Body-Le corps en scène 2002, p. 87). Prière de lire à ce sujet : Walker 1987.

25 Plougastel 1997, p. 3.

26 Soucy Automne 1999, p. 29.

27 Cf. Griffiths 1995, p. 174. 
Zappa $^{28}$, Brian Eno, Philip Glass ${ }^{29}$, Michael Gordon, Gavin Bryars, Michael Daugherty, Georges Aperghis ${ }^{30}$, Aaron Jay Kernis ${ }^{31}$, Heiner Goebbels ${ }^{32}$, Lorenzo Ferrero, Graham Fitkin, Alejandro Viňao ${ }^{33}$, Philippe Hurel, Antonio Victorino d'Almeida, Laurie Anderson ${ }^{34}$, Pascal Zavaro, James Giroudon, Pierre-Alain Jaffrenou, Jonathan Pontier ${ }^{35}$, Sébastien Gaxie, Fred Griot ${ }^{36}$, Nicolas Tzortis, Casper Cordes... et bien d'autres ont été marqués par «l'idéologie de l'authenticité37 » du Rock ou, plus largement, ont été imprégnés de l'esprit Pop made in $U_{S A}{ }^{38}$ (au reste, dans le dédale catégoriel des appellations, SophieAnne Leterrier remarque qu' «en France, le rock peut être qualifié de musique pop, tandis qu'en Grande Bretagne la notion de pop music est plus liée à l'uni-

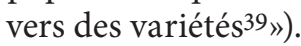

Pour mémoire, mode d'expression «branché» des jeunes $4^{40}$ dès le début des années 1950, le Rock a vu ses premiers succès se couronner grâce à Good Rockin' Tonight (1948) par Ray Brown, puis à Crazy man crazy (1953) et Rock around the Clock (1955) par Bill Haley... pour finalement arriver en France à partir de 1956. Moins d'une décennie plus tard, plébiscités à l'envi par la jeunesse avide de sensations aigres-douces, The Beatles ${ }^{41}$ ont vu leurs volumineux impacts s'ancrer au sein de pièces savantes ${ }^{42}$ signées par exemple par Cathy Berberian

28 A propos de Frank Zappa qu'il estimait (et avec qui il a collaboré), Pierre Boulez a noté que « celui qui cherche vraiment à s'exprimer par l'art doit être capable de se confronter aux niveaux les plus élevés de connaissance, de technique, de réflexion, parce que, de ce point de vue, une hiérarchie reste valide. " (Boulez 9 mars 2001, p. 12).

29 Dans un article du Monde paru le 21 octobre 1976, Daniel Caux a noté que le courant "répétitif » américain pouvait, de près ou de loin, évoquer le jazz ou le rock (propos repris dans Caux 2009, p. 73). Pour mémoire, Philip Glass a notamment écrit Heroes Symphony d'après des musiques de David Bowie et Brian Eno ainsi que des songs avec la collaboration de Paul Simon et Laurie Anderson. Au début des années 1980, il a même produit le groupe de musique New-wave Polyrock. À l'inverse, l'art de Phil Glass influencera la musique de David Bowie, de Brian Eno ou de Yann Tiersen, de Kraftwerk ou de Cluster. Par ailleurs, ancien batteur, Steve Reich qui s'est passionné pour les percussions du Ghana et de Bali n'a-t-il pas été honoré à son tour par DJ Spooky et par eRikM ? Avec ces divers exemples, il est aisé de montrer que l'alternance et l'échange sont récurrents au cœur des sphères musicales savante et populaire.

30 Auteur de Rock'n roll (1984) pour voix et ensemble, partition extraite du Bal de la Contemporaine.

31 Cf. Brackett 2002, p. 215.

32 Ex rocker du groupe expérimental Cassiber, Heiner Goebbels a composé en 1980 un Rock gegen Rechts pour piano et saxophone ténor.

33 Cf.Collins et d’Escriván 2007, p. 187.

34 Cf. Rockwell 1985, p. 130.

35 Cf. Ravet 2010, p. 27-40.

36 Auteur du spectacle pluridisciplinaire Parl \# (2011) pour voix, guitare et batterie à l'énergie rythmique puisée dans le monde du Rock.

37 Sibilla 2003 , p. 23.

38 Cf. Castanet 1998, p. 1110-1116.

39 Leterrier 2010, p. 199.

40 Le « coup de jeune » des amateurs de pop s'amplifiera dans les sixties. En effet, « la « jeunesse ", dans les années soixante, est devenue un mythe, incarnation à la fois d'une modernité dynamique et symbole de la culture de masse, à admirer et à craindre ", analyse Pascale Goetschel (cf. Goetschel 2010, p. 42).

41 Cf. Holmes 2008, p. 407-411.

42 Cf. Castanet 1999 (rééd. 2007), p. 60. 
(dans Stripsody - 1966 - citant allègrement She's got a Ticket to ride) ${ }^{43}$, par John Cage ou Michael Chant... Même si l'iconoclaste pianiste Glenn Gould signale que «sur le plan tonal, les Beatles éprouvent aussi peu de considération pour les raffinements de la polyphonie qu'Erik Satie en manifestait pour les entrecroisements harmoniques éperdus des post-romantiques allemands 44 », évoquons ces multiples arrangements de Hey Jude et autre Michelle signés par Leo Brouwer, Toru Takemitsu, Louis Andriessen, Luciano Berio, Pauline Oliveros, Michael Finissy, Kaija Saarihao, Sigmund Krauze, Terry Riley, Alvin Lucier ou Christian Rivet ${ }^{45}$....

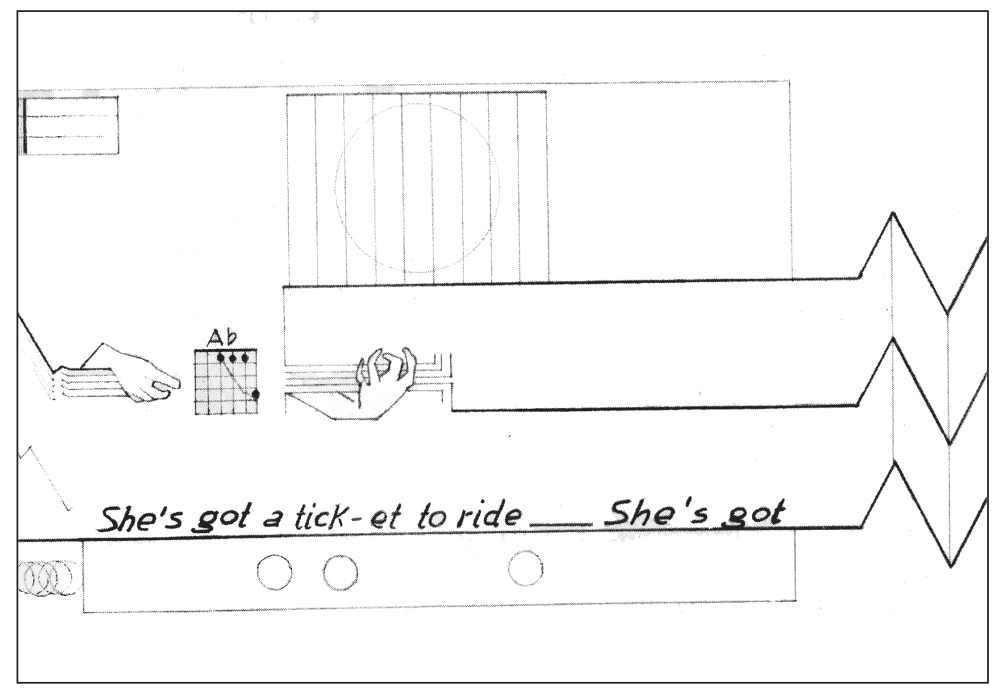

Exemple A: Extrait de Stripsody. Partition graphique pour voix seule de Cathy Berberian. (New York, Éditions Peters no 66164, graphique de Roberto Zamarin, 1966, p. 8)

De plus, sans parler des citations des chansons des Beatles incrustées subrepticement ou pas dans la chair du discours savant (Per Norgard dans Tango Chicane ou dans I Ching, Bernd Alois Zimmermann dans Requiem pour un jeune poète, Aldo Clementi dans Collage 2, John Cage dans The Beatles 1962-1970, Constança Capdeville dans Take 91...), des phalanges de musique contemporaine ont incorporé (à l'image de Mark-Anthony Turnage vis-à-vis des jazzmen) des rockers à leur formation. Voyez l'équipe italienne de Musica Elettronica Viva de Frédéric Rzewski et Salvatore Esposito ou l'ensemble américain Theatre of Eternal Music de La Monte Young invitant par exemple le rocker gallois John Cale ${ }^{46}$ à venir jouer parmi les musiciens dits «sérieux» du collectif. À l'inverse, le New York Times nous a appris en 2007 que le percussionniste

43 Cf. MagnifiCathy - The many voices of Cathy Berberian (1928-1983) 1988.

44 Gould, Monsaingeon 1985, p. 442.

45 Rivet 2009.

46 Influencé par John Cage, John Cale a collaboré avec La Monte Young. En compagnie de Lou Reed, il a fondé le groupe The Primitives puis le célèbre Velvet Underground produit par Andy Warhol. 
Ted Atkatz avait quitté le Chicago Symphony Orchestra pour entrer en tant que batteur dans un groupe de rock alternatif47. Dans toutes ces circonstances où l'esprit de pluralité dominait, l'idée de fusion des sources planétaires était déjà en germe ${ }^{48}$, tentant d'accéder aux vœux postmodernes de l'universitaire Remo Ceserani demandant à la critique de «cesser de soutenir la distinction entre les arts élevés et les arts de masse 49 » [...].

Parmi maints exemples qui nous conduiraient jusqu'au genre équivoque de l'opéra-rock (de Freak out ! - 1966 de Frank Zappa - à Dracula - 2011 - en passant par Tommy - 1969 -, Jesus Christ Superstar - 1970 -, Excalibur 1998 -, Roméo et Juliette - 2000 -, Mozart - 2009 - et bien d'autres $)^{50}$, et en dehors de terrains « de contestation à la fois sociale, culturelle et idéologique ${ }^{51}$ ", le médium du Rock (ou de la chanson pop) ne s'est pas seulement lové - avec une certaine logique ${ }^{2}$ - au travers des clichés people liés aux valeurs hasardeuses d'une antique vérité à survoler de loin ou au cœur des spots publicitaires terriblement aguicheurs - mais la plupart du temps tellement kitsch - à destination de l'audimat des radio, télévision ou web, il s'est également niché jusque dans les plis et les replis de véritables partitions de musique contemporaine.

Ainsi, de l'aveu de John Adams, le début de sa partition intitulée Naive and Sentimental Music (1997-98) est fondé harmoniquement sur un modèle de songs légué par Joni Mitchell ou Paul McCartney53. Traitant de la dichotomie de l'«objet esthétique» et de l'« œuvre d'art», Marie-Claude Lambotte a insisté sur «la composition des signes appelés à configurer la chose (ce que le philosophe désignerait par la notion de "style») comme une composition qui n'est pas donnée en soi et qui demanderait une sorte d' "acquiescement» en vue de son appropriation par le sujet ${ }^{54}$ ». En l'occurrence, l'esprit du Rock a ceint autant l'aura de son instrumentarium à fort dégagement "décibellique» que le statut bigarré de l'hybridation sonore y afférant. Se déroulant en 1968, l'opéra Jackie $O$ (1993) de Michael Daugherty55 - qui met en scène les personnages de Maria Callas, Andy Warhol, Liz Taylor, Grace Kelly, entre autres - a ainsi mixé au discours musical savant moult sources provenant des mondes de la comédie musicale, de la chanson, du jazz et du rock ${ }^{56}$.

47 Wakin 2007.

48 À l'inverse mais toujours dans ce sillage de la mixité, certains groupes ont flirté avec la musique classique: Aphrodite's Child, The Beatles, Deep Purple, Walter Carlos, Yes, The Nice, Magma, The Yardbirds, Emerson, Lake and Palmer... (cf. Castanet 1999 (rééd. 2007), p. 132).

49 Ceserani 1997, p. 32 (notre traduction).

50 Qu'elles soient en rapport avec les idoles ou les figures légendaires (Roméo et Juliette, Dracula), la référence historique à la royauté (Le Roi Soleil) ou au Messie (Jesus Christ Superstar) comme la révérence à l'auteur des Noces de Figaro (Mozart-spectacle mi-classique, mi-Rock qui existe également en film 3 D) montrent à l'envi que « le passé tout entier rentre dans le circuit de consommation " (cf. Baudrillard 1968, p. 102).

51 Tamagne 2010, p. 718.

52 Cf. Jameson 1984.

53 Cf. Marshall 2002, p. 5.

54 Lambotte 2003, p. 155.

55 Ex batteur et pianiste de jazz, fondateur d'un groupe de Funk (cf. Lelong 1996, p. 98). Ce compositeur américain (né en 1954) a été l'élève d’Earle Brown et de György Ligeti, ainsi que le collaborateur du jazzman Gil Evans.

$56 C f$. DVD Dynamic n ${ }^{\circ} 33605$ réalisé en 2009. 


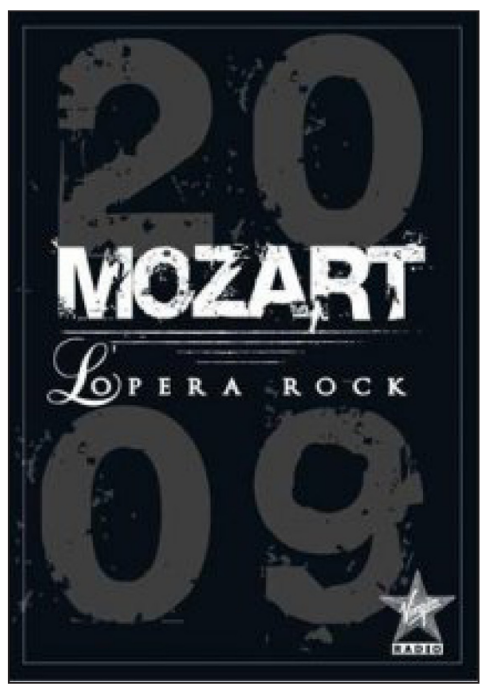

Figure 1.

Subodorant les prodromes d'une «nouvelle sensibilité57», Luciano Berio qui comme György Ligeti appréciait la musique des Beatles - a ainsi comparé, en mai 1967, le son du Rock manipulé d'une manière électronique à celui de la musique savante fabriqué dans les studios de Cologne ou de Milan, même si, selon le compositeur italien, il est arrivé «que le rock, comme la musique électronique - tous deux créatures de la radio et de sa machinerie de masse - soient paradoxalement incompatibles avec les moyens de diffusion qui en ont provoqué le développement ${ }^{58}$ ». Partiaux ou sectaires, les annotateurs plus ou moins avisés ont souvent constaté que la flèche du temps a modifié à chaque époque le sens directionnel de la modernité, l'artiste d'avant-garde étant «celui qui s'invente une nouvelle généalogie, qui tisse une nouvelle "intrigue» dans l'histoire de l'art, qui conteste, et souvent violemment, certaines idoles qui dominent la scène culturelle ${ }^{59}$ », tient à distinguer à sa manière Martin Kaltenecker.

\section{ÉTUDES DE CAS : QUELQUES «SCENARIOS » MYTHOLOGIQUES CONTEMPORAINS}

Alors que pour Roland Barthes, en 1956, «le mythe est une parole ${ }^{60}$ », nous avons tendance à orienter nos propos - depuis une quinzaine d'années en montrant et démontrant qu'il est figuré également par l'aura exclusive du tout-sonore ${ }^{61}$. Dès lors, si le son du Rock est considéré comme médium, il peut

57 Termes militants de l'époque chers à Suzan Sontag, (cf. Sontag 1966).

58 Berio mai 1971, p. 59 (pour la version en langue française).

59 Kaltenecker 2000, p. 3.

60 Barthes 1957, p. 193.

61 En dehors de Tout est bruit pour qui a peur-Pour une histoire sociale du son sale, op. cit., se référer entre autres à : Quand le sonore cherche noise-Pour une philosophie du bruit; "Pour une 


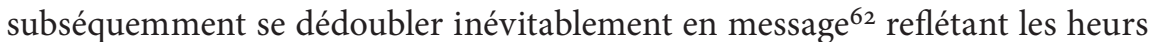
et surtout les malheurs de la comédie humaine. Alors que nous avons le son que nous méritons, les avatars «bruiteux» du concert contemporain nous renvoient tantôt à quelques divertissements à vocation plus ou moins déterministe (habitus) ${ }^{63}$, tantôt aux divers conflits larvés minant sporadiquement les affres de notre société (male habitus) ${ }^{64}$.

Afin de compléter les liens déjà mentionnés dans la première partie, les quelques études de cas qui vont suivre désirent exposer les différents rapports du Rock avec le monde de la musique contemporaine, en jouant de la proximité intrinsèque du fond comme de la métaphore de la forme. Rappelons que selon le théoricien de la communication Marshall Mac-Luhan, le vrai message ne réside pas dans son contenu même mais dans la façon dont il est transmis (son medium). Englobant la pluralité des signifiants, des signifiés et des signes, ce dernier va permettre de véhiculer rapidement à un très grand nombre de personnes les éléments d'une philosophie, d'une tendance, d'un genre, d'un style, d'une manière d'être ou les modes d'appréhension des vicissitudes de la vie... En l'occurrence, le médium du Rock a pu aisément s'infiltrer au travers des parois poreuses de la culture globale et ainsi influencer fortement le récepteur (l'illettré de la campagne comme l'étudiant du conservatoire). Accusé par la technique itérative du matraquage médiatique ${ }^{65}$, cette forme de médiologie à l'emporte-pièce va ainsi modifier - à l'insu ou pas de l'auditeur passif ou actif - sa façon de penser, de se comporter, mais aussi de concevoir des règles hybrides inédites ou de nouveaux modèles équivoques pour un autre monde sensoriel, somme toute globalement métissé.

Dans la Messe pour le temps présent (1967) coréalisée avec Michel Colombier ${ }^{66}$, Pierre Henry a par exemple introduit un Psyché Rock volontairement stylisé (étiqueté même par certains commentateurs de «bal de province») ${ }^{67}$. Composé pour les ballets de Maurice Béjart, ce morceau d'anthologie, zébré par des salves électroniques incongrues pour l'époque, s'est hissé à la première place du hit-parade français (enregistrement sorti à l'époque en disque 45 tours). Non conforme aux règles de caste, il a contribué à l'abattement des frontières entre les sphères savante et populaire ${ }^{68}$. Au même moment, Jerry Rubin - leader du mouvement Hippie - élaborait aux États-Unis quelques "scénarios de la révolution par le rock»: «Elvis bousilla l'image papa-gâteau

bruitologie performantielle »; « Regards sur la première décennie du XXIème siècle : pour une " sonodoulie " complexe aux allures décomplexées "; " De la compromission identitaire de la musique contemporaine à l'époque postmoderne »; "Les grimoires de Mnémosyne : pour un palimpseste des origines »; " "Ouvrir son cœur à la création" : notes sur l'art du ready-made sonore ».../...

62 Mac-Luhan 1967.

63 Cf. Bourdieu 1980.

64 Cf. Castanet 1999 (rééd. 2007), p. 17.

65 Mac-Luhan 1968.

66 Compositeur de musiques de film, Michel Colombier (1939-2004) a été l'arrangeur de chansons cultes de Claude Nougaro, Barbara, Brigitte Fontaine, Serge Gainsbourg, Madonna...

67 Cf. Castanet 1999 (rééd. 2007) p. 100.

68 Pierre Henry réitérera son flirt avec la pop music en composant en 1969-70 une « messe électronique » intitulée Ceremony avec la participation du groupe anglais Spooky Tooth dont le leader et chanteur est Gary Wright. 
d'Eisenhower en secouant à mort nos jeunes corps emmaillotés. L'énergie sauvage du rock gicla en nous, toute bouillante et le rythme libéra nos passions refoulées ${ }^{69}$ ", pouvait-il témoigner à chaud dans son manifeste baptisé Do it. Au milieu des années 1930, Denis de Rougement avait déjà pensé que «la mission de la culture est de conduire une révolution qui, sinon, se fera contre elle ${ }^{70} \%$.

Hormis Pierre Henry, d'autres membres du Groupe de Recherche Musicale (GRM) à Paris ont rendu hommage à la musique populaire de la fin des sixties. Signalons par exemple Bernard Parmegiani avec Du pop à l'âne (collage contenant des musiques pop superposées-1968) et Pop'eclectic (d'après une musique de film aux sons réalistes et aux accents parfois hollywoodiens-1969). Mis à part le passage du jeune Jean-Michel Jarre dans cette noble institution ${ }^{71}$, citons plus sérieusement François Bayle avec la pièce caudale de La Preuve par le sens (1971) où par le truchement d'un geste d'autocitation (Solitioude-1970), l'oreille attentive peut entendre en arrière-fond des agissements vocaux improvisés par le célèbre batteur Robert Wyatt ${ }^{72}$ sur I did it again. Dans le même intervalle, au niveau du jeu inopiné, distinguons aussi l'exemple de la communauté singulière du groupe italien Musica Elettronica Viva dont les fortes personnalités (Allan Bryant, Alvin Curran, Fredric Rzewski, Richard Teitelbaum...) prenaient un malin plaisir à combiner allégrement bandes magnétiques, lutherie électronique et instruments acoustiques, sources sonores primitives et bribes de musique orientale, ready-mades amplifiés (objets du quotidien) et sons organiques, mélismes mélodiques empruntés au Jazz et reliefs rythmiques provenant du Rock.

En France, remarquons Randon Access Memory de Tristan Murail débuté en 1984, partition qui se présente comme une «Rock-Messe» écrite pour sextuor mixte: guitares électriques, batterie, percussion, synthétiseurs et ordinateurs. Ondiste de formation, ce compositeur - membre fondateur de l'ensemble L'Itinéraire (Paris) - a été attiré au travers de la mouvance soixante-huitarde européenne par le pool électronique émanant des orchestres de "Kosmische Musik» (comme on disait à l'époque à Berlin-Ouest) et par les sonorités de Jazz-Rock progressif73. Désirant œuvrer sur l'hybridation entre expression rock et langage musical savant, cet élève d'Olivier Messiaen a expliqué que l'on pouvait trouver « des points de rencontre entre ces deux univers a priori si différents, dans l'utilisation de certains processus, dans le domaine des rythmes ou des harmonies, mais surtout dans le domaine des timbres. La musique joue ainsi sur les ambiguïtés créées par l'exploitation des domaines communs à ces deux mondes musicaux ${ }^{74}$ ». In fine, à l'écoute de Randon Access Memory, la critique hexagonale décela l'influence des Pink Floyd75.

\footnotetext{
69 Rubin 1971, p. 18.

70 de Rougemont 1972, p. 195.

71 Cf. Kyrou 2002, p. 62.

72 Marxiste révolutionnaire, Robert Wyatt a été le leader du groupe Soft Machine, formé en 1966.

73 Cf. Castanet 1999 (rééd. 2007), p. 102.

74 Tristan Murail cité par: Thierry Alla (Alla 2008, p. 152).

75 Cf. Le Républicain lorrain du 5 octobre 1984.
} 
Dans le sixième mouvement de l'œuvre ${ }^{76}$ intitulé Vampyr ! (1984), Tristan Murail confie à une guitare électrique (accompagnée d'une guitare basse ad libitum dans la seconde partie) un son proche de ceux crissant des "guitarheros» (Carlos Santana ou Eric Clapton). En l'occurrence, le jeu demandé doit générer une aura sonore mouvante émanant d'un geste digital souple qui favorise naturellement les micro-intervalles. "Il s'agit plus de retrouver le son saturé des amplificateurs à lampe que d'obtenir une vraie distorsion, trop proche du bruit», explique avec fougue et détermination le compositeur. Il faut «faire passer dans Vampyr ! toute l'énergie de la musique rock, avec le nombre de décibels appropriés!», conclut-il77. Sur la partition, on lit que Tristan $\mathrm{Mu}$ rail désire entendre "une véritable guitare électrique de type "Rock", munie d'une manette de vibrato (indispensable !), jouée impérativement au plectre, une pédale de distorsion du type saturation d'ampli à lampes, une pédale de volume et un ampli» (ad libitum un flanger stéréo pour un léger reflet "chorus" et d'espace, un deuxième ampli pour la stéréo $)^{78}$ \%.

Durant les quatre mesures présentées dans cet exemple, en dehors des coups de médiator notés au-dessus des mi graves (corde à vide), on reconnait la technique de glissando des rockers réalisé avec la manette de vibrato (notée «B »). Au reste, Thierry Alla est d'avis que cette pièce de Tristan Murail pourrait s'intégrer facilement à un véritable concert de Rock'n roll. «Le son saturé est très proche du fameux solo improvisé sur l'Hymne américain de Jimmy Hendrix au festival de Woodstock en 1969. [...] Le travail sur le timbre est surtout construit à partir des effets 79 ». Il faut dire que, passant outre les mots d'ordre esthétiques et les modes passagères, Tristan Murail a toujours œuvré dans la conceptualisation inédite de mécanismes syntaxiques de simulation, de différenciation, d'intégration, de régulation, de sélection, de réverbération, de spatialisation, de formalisation et d'informatisation... Et s'il a versé dans la systématisation de principes (de la modulation en anneau à la création de spectres inharmoniques, en passant par l'usage de la boucle, de la distorsion, de l'écho, du filtrage, du phasing...), il a également mis en place les éléments typés d'une poétique dialectique visant le matériau sonore et sa propre régie de force interne, donnant corollairement et naturellement naissance aux fameux processus (interrelationnels) de forme mouvante et de flux dynamique continu ${ }^{80}$.

Nous pourrions aussi évoquer la part de «tradition de violence, de révolte et de sexualité exacerbée» qui alimente le «vieux rock $^{81}$ » et celle complémentaire du «vacarme assourdissant ${ }^{82}$ » qui couronne tout Rock-Festival digne de ce nom. Dans ces conditions comportementales et environnementales, le deuxième aspect attirant relevé par Luciano Berio au sein de ses "Commentaires au rock» concerne le domaine de la vocalité (son naturel, sa spontanéité,

\footnotetext{
76 Euvre qui comporte neuf mouvements en tout.

77 Murail 1987, p. 2.

78 Cf. Murail, ibidem.

79 Alla 2008, p. 155.

80 Cf. Castanet (préface Alla 2008, p. IV).

81 Bizot mai 1971, p. 77.

82 Morin 1970, p. 114.
} 


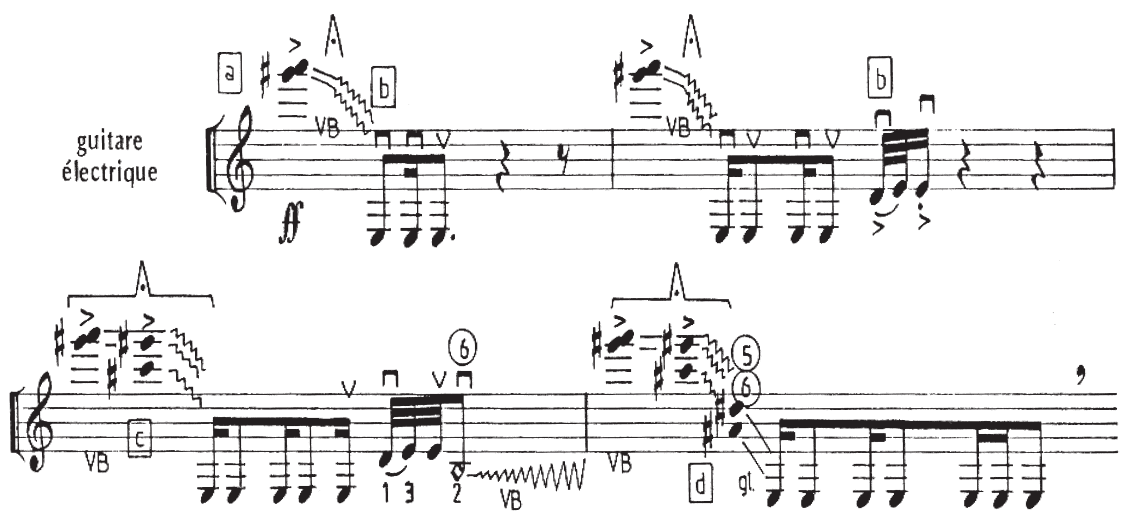

Exemple B : Début de Vampyr ! pour guitare électrique de Tristan Murail. (Paris, Éditions Salabert, 1985 , p. 8)

son expression plurielle). Ainsi, «la plupart du temps, la voix crie, c'est vrai, mais chacun crie à sa manière, sans affectation. De plus, les textes, lorsqu'ils ne sont pas réduits à des «rituels" verbaux, à des nonsense ou à des onomatopées instrumentales sont rarement stéréotypés et sont peu redondants (une certaine redondance est de toute façon fournie par la forme répétée de presque toutes les chansons rock) ${ }^{83}$ », analyse le maestro.

En outre, au cœur des différents chapitres de cette histoire sociale de la musique $^{84}$ et une fois pris en compte le domaine de la langue anglaise qui favorise allègrement le jeu syncopé ${ }^{85}$ du relief mélodique, l'idée du cri émergeant de la complexité ambiante reste bien la métaphore énergique ${ }^{86}$ et acoustique, éhontée et agressive ${ }^{87}$ du Rock. Dans un credo anecdotique, le compositeur Nicolas Tzortzis ${ }^{88}$ se prononcera plus tard en disant qu'il «aime depuis toujours la musique qui a de l'énergie, comme le $\operatorname{rock}^{89}$ » et que cette influence se retrouve indirectement dans ses partitions sérieuses. En tant que «chaos musical de l'âme ${ }^{90} »$ ou en tant qu' «appel abyssal ${ }^{91} »$, cette image sonore signalétique sera

83 Berio 1971, p. 59
84 Cf. Frith 1978.
85 Cf. Ligeti 2001, p. 15 .
86 « Convoquer le monstre chtonien existant en chacun, exprimer le mal (c'est-à-dire le faire sortir de soi), exalter l'excès, sont des manières de trouver ou de retrouver de l'énergie. Ainsi l'orgiasme musical et les drogues lui servant d'adjuvant sont une méthode tragique de crier et de vivre l'éternité. Une éternité immanente, enracinée dans l'ici et maintenant» (Maffesoli 2008, p. 198).

87 «Le rock'n'roll était une musique tout ce qu'il y a de plus simple. L'important, c'était le bruit que ça faisait, son énergie, son agressivité, sa nouveauté. Le seul tabou, l'ennui », témoigne Nik Cohn (Cohn 2004, p. 37). De même, cette fois dans le domaine savant, Daniel Caux avait remarqué que l'univers sonore de Louis Andriessen s'acoquinait volontiers avec «l'agressivité du Rock » (cf. Caux 2009, p. 146).

88 Né en 1978 à Athènes, ce compositeur a été l'élève de Philippe Leroux, Georges Aperghis, Horacio Vaggione et Manuel López López.

89 Tzortzis 2010, p. 113.

90 Expression de Darbon 2006, p. 38.

91 Quignard 1996, p. 130. 
à retrouver dans les vociférations d'Iron Maiden ou les improvisations de Joëlle Léandre, dans les mises en scène bruyantes de la fluxiste Yoko $\mathrm{Ono}^{92}$ ou dans les expérimentations de Meredith Monk, dans l'attitude sonique des Pink Floyd (The Wall) ou dans la vidéo démonstrative d'Absalon intitulée Bruits $(1993)^{93}$, dans les solipsismes vocaux demandés par Giacinto Scelsi (au sein des Chants du Capricorne) ou dans les relations à la folie illustrées magnifiquement par Sir Harrison Birtwistle et par Wolfgang Rihm, voire dans les répliques déchirantes de free jazz léguées par Max Roach et Abbey Lincoln ${ }^{94}$.

Car l'utilisation de l'extra-vocalité (cris, râles, pleurs, rires...) 95 comme des effets pesants ${ }^{96}$ de "son sale» (Larsen, Fuzz bass, pédale Taurus ${ }^{97}$, amplifications saturées, sonorités psychédéliques, "gadgets ${ }^{98}$ » divers) présente les signes identitaires d'une émancipation culturelle 99 et en même temps d'un affranchissement vers un territoire emblématique, voire initiatique. Ainsi, «dans les sociétés où l'accession à la condition adulte n'est ni réglée ni encadrée, les adolescents tendent spontanément à satisfaire leurs besoins les plus profonds en forgeant un milieu culturel spécifique dont les manifestations sont à rapprocher des rites de passage des sociétés primitives ${ }^{100}$ ». Louvoyant entre expression indistincte et discours intelligible - et dans un contexte parfois Rock-, René Lussier (Vive le Québec libre par le Général de Gaulle) ${ }^{101}$ comme Hermeto Pascoal (Tiruliruli, Vai mais Garotinho) ont tenté de sauvegarder cette forme de «trésor de la langue» en s'inspirant des techniques de Speech Rythms inaugurées par Frank Zappa, c'est-à-dire en accusant le relief mélodique de l'oralité publique (d'hommes politiques comme de speakers sportifs). De même, entre art savant - épris de structures varésiennes et de rythmiques stravinskiennes - et expression populaire débouchant sur la chanson, Zappa (instrumentiste, compositeur, acteur, réalisateur, producteur) a fortement brillé par ses bigarrures, son éclectisme, son "sens de l'ironie, son goût de l'absurde, son cynisme réjouissant, la bizarrerie de ses constructions, ses commentaires sarcastiques autant que lucides ${ }^{102} »$.

92 Cf. Ono 2004, p. 26.

93 Cf. Electric Body-Le corps en scène 2002, p. 130.

94 Cf. Triptych: Prayer / Protest / Peace enregistré en duo en 1960 (cf. CD We insist).

95 Éléments figurant au cœur de la sixième catégorie de bruits de l'orchestre futuriste proposée par Luigi Russolo dans son manifeste intitulé L’Art des bruits (Russolo 1975, p. 41).

96 On songe à « la voix nasillarde et jamais en place de Mick Jagger », aux «notes sales », au « son dur, lourd et redondant des Rolling Stones, qui créent sur disque comme sur scène, dans leur meilleure période, un climat d'insécurité permanent » (Yonnet 1985, p. 161).

97 Cf. Pirenne 2005, p. 121 et p. 214.

98 «Pédale Wawa, Fuzz, distorsion, compression, cellules d'amplification des cuivres, cerclages micros des batteries, orgues électroniques, mélotron, Moog », repère Alain Roux au début des années 1970 (Roux 1973, p. 131). Lire également Rollin 1994, p. 60-61. Afin de saisir concrètement les effets de la pédale Wawa, écoutez par exemple : Electric Ladyland de Jimi Hendrix ou Willie the Pimp de Frank Zappa...

99 Cf. Hall et Du Gay 1996.

100 Bloch et Niederhoffer 1984, p. 29.

${ }^{101} \mathrm{Cf}$. CD Le Trésor de la langue 1989.

${ }^{102}$ de Caunes 2010, p. 689. 
De plus, adepte d'une certaine forme d'«extraphonie ${ }^{103 », ~ F r a n k ~ Z a p p a ~-~}$ l'auteur de Sheik Yerbouti aux accents caricaturaux de Rock - ne demandait-il pas d'être «réaliste»? Car «la guitare peut-être l'objet le plus violent du monde. C'est pour ça que je l'aime... Le son dégoûtant d'une guitare électrique trop forte: c'est ce que j'appelle passer un bon moment», déclarait-il à un journaliste de Sound International en avril $1979^{104}$. À la suite, le guitariste Steven Mackey s'inspirera de ces effets noisy dans le symbolique Grungy (1994) écrit pour guitare électrique. De même, déconstruites puis réassemblées en studio, les interventions sauvages d'un Fred Frith (par exemple dans Image in and Atom ou dans Touch I Risk enregistrés en 2001) se positionneront dans la filiation de cette quête de son perverti par les soins de la Fée électricité (univers improbable allant de la guitare MIDI au koto électronique). Il en sera de même pour les partitions agressives de Jimi Hendrix, de Lou Reed ou de Robert Fripp. À propos de toutes ces musiques populaires où le sound est fabriqué sur mesure ${ }^{105}$ pour attirer le plus de fans aux aguets et être reconnaissable dès les premières secondes du jeu, mentionnons également l'opinion de Brandford Marsalis: «Ce que j'aime dans le hip-hop - si c'est de la bonne musique - , c'est qu'il possède un son vraiment fort ${ }^{106}$ ».

\section{L'APPROPRIATION DE L'INSTRUMENTARIUM ROCK}

Envahissant et personnalisé, si le Rock peut représenter à la fois «une figure aux aspects restrictifs de ses descendances stylistiques et un hommage aux forces libératrices de l'éclectisme» - comme l'explique Luciano Berio -, il rayonne également grâce à son aura timbrique et son énergie dynamisée. Ainsi, cette musique a-t-elle «les moyens de noyer tout cela dans un bruit plus infernal, mieux organisé ?107 ", se demandent les auteurs de Rock babies. Miroir à facettes du «Tout-Monde ${ }^{108}$ », «tout comme la philosophie, le rock nous aide à comprendre le monde et à mieux vivre ${ }^{109}$ ", commente Francis Métivivier. Le troisième argumentaire concerne à n'en point douter la nature (électro)acoustique de la lutherie ${ }^{110}$ en présence: «Ils ont un son différent», disent fréquemment les uns des autres, les musiciens appartenant à des groupes divers, la typologie sonore étant l'unique constante qui permette d'évaluer des différences "linguistiques"111», souligne encore le compositeur italien.

Traitant du «syndrome de l'imitation», Jean-Claude Klein écrit: «Là où la génération 1960 aspirait à s'exprimer avec une caméra ou une guitare acoustique, et celle de 1968, avec une ronéo, celle des années 80 choisit de se faire

\footnotetext{
${ }^{103}$ Cf. Castanet 2008, p. 54-55.

104Cf. Bacon 2004.

${ }_{105}$ Cf. Castanet 2008, p. 286-29o.

${ }^{106} \mathrm{Cf}$. Rousselot 1995.

107 Hoffmann et Leduc 1978, p. 190.

${ }^{108}$ Cf. Glissant 1997.

109Métivivier 2011, p. 4.

110 Pour information : $1^{\text {ère }}$ guitare électrique en 1931, 1 ère guitare basse électrique produite en série en $1951,1^{\text {er }}$ solo de batterie en studio en $1962,1^{\text {ère }}$ pédale wah-wah pour les guitares électriques en $1967,1^{\mathrm{er}}$ synthétiseur en 1967... (cf. Quint 2010-2011).

111 Berio 1971, p. 58.
} 
entendre avec une sono, une guitare et une batterie ${ }^{112}$ ». C'est à travers ce prisme que les instruments et la technologie ${ }^{113}$ de l'orchestre Rock (usage de Mellotron, orgue Hammond, Moog, Crumar, Farfisa FAST, Fender Rhodes et autres synthétiseurs.... ${ }^{114}$ ont été sciemment récupérés et abondamment utilisés par les compositeurs de musique contemporaine, même si comme le rappelle Tristan Murail, «les fabricants de synthétiseurs, amplificateurs, etc., ont défini leurs produits en fonction des besoins de la musique populaire ${ }^{115}$ ». Dans un manifeste réunissant les idées-forces du CRISS (Collectif de Recherche Instrumentale et de Synthèse Sonore - Paris), Hugues Dufourt clame en 1978 que le "son électrique suscite un langage nouveau ${ }^{116}$ ». Pour sa part, le compositeur Sébastien Béranger déclare que «la plupart des outils utilisés en musique contemporaine étant développés a priori pour les mass media (radio, TV, musiques dites «de variété» et surtout cinéma), ils ont une influence certaine sur la création - de la conception à la réalisation finale ${ }^{117}$ ». Orgues, guitares électriques, guitares basses et batteries seront donc de la partie.

\section{L'ORGUE ÉLECTRIQUE}

Traité en soliste dans la Messe pour le temps présent (1967) de Pierre Henry et Michel Colombier (œuvre déjà mentionnée), l'orgue électrique a souvent été couplé avec des registres d'instruments à vent pour ses qualités d'amalgame empirique mais probant d'harmoniques naturels, différentiels ou agrégatifs. Enluminé par Rhoda Scott, Sun Ra, Eddy Louiss, Stevie Wonder, Ray Manzarek des Doors, Ian Stewart des Rolling Stones ou Stephen Stills du quatuor Crosby, Stills, Nash and Young, et éminemment présent au cœur des albums de Delaney \& Bonnie ou des Beach Boys..., cet instrument à clavier aux mille couleurs dégradées a séduit autant Friedrich Cerha (Mouvements I-III-1960) que Mauricio Kagel (Tremens - 1963-65), Giacinto Scelsi (Hymnos - 1963, Konx-Om-Pax - 1969, Pfhat - 1974), Karlheinz Stockhausen (Mikrophonie II-1965), Louis Andriessen (The Garden of Ryoan-gi - 1967), Phill Glass (One plus one - 1968, Einstein on the Beach - 1976), Luciano Berio (Sinfonia 1968), Hugues Dufourt (Mura della Città di Dite - 1969, Dusk-Light - 1971), Tristan Murail (Couleur de mer - 1969), Steve Reich (Four Organs - 1970, Phase Patterns-1970, Music for Mallet Instruments, Voices and Organ - 1973), Salvatore Sciarrino (Un'imagine di Arpocrate - 1974-79), György Ligeti (Le Grand Macabre-1975-76), Wolfgang Rihm (Faust und Yorick - 1976), Gérard Grisey (Sortie vers la lumière du jour - 1978, Jour, contre-jour - 1978-79)... pour ne parler que des années $1960-70^{118}$.

112 Klein 1984, p. 186-187.

113 Cf. Bacon 1981.

$114 C f$. Pirenne 2005, p. 270, 273, 279, 280, 283... À propos de la « nouvelle lutherie », prière de lire :

Lemery mai 1971, p. 84 .

115 Murail 2001, p. 60.

${ }^{116}$ Cf. Texte du manifeste reproduit dans : Castanet 1995, p. 322.

117 Béranger 2006, p. 41.

118 Pour en savoir plus, prière de consulter : Louvier et Castanet 1997, p. 107-108. 


\section{LA GUITARE ÉLECTRIQUE}

En dehors de Tristan Murail dans Vampyr! (solo déjà cité), signalons pour mémoire Francis Miroglio dans Tremplins (1968), Hugues Dufourt dans Saturne (1978-79) ${ }^{119}$, Alain Bancquart dans sa Première symphonie (1979) ${ }^{120}$, Rhys Chatham (ancien hard-rocker) ${ }^{121}$ dans Warehouse of Saints, Songs for Spies ${ }^{122}$ (1989-91), Roger Tessier dans Scène VI - Au rivage, comme un navire (1994-95) et dans Fissure(s) (2011), Philippe Manoury dans l'opéra 6o ème parallèle (199798) ${ }^{123}$, François Bousch dans Fissions d'échos (1986), Scott Johnson dans Rock/ Paper/Scissors (1990-94), Steven Mackey dans Grungy (1994), Richard Barrett dans Transmission (extrait de Dark Matter - 1996-99), Claude Ledoux dans Zap's Init (2008), Gavin Bryars dans De la crudel morte de Cristo - Lauda 41 (2011), Mark-Anthony Turnage dans son opéra Anna Nicole (2011)... De générations et de cultures différentes, ces compositeurs européens ont laissé sonner à foison les combinaisons les plus saisissantes (parfois gorgées de larsen) et résonner les captivants effets couplés à une écriture complexe et souvent virtuose de l'instrument à six cordes électrifié de Jimmy Hendrix. Par extrapolation, car vu sous un angle purement philosophique, Vladimir Jankélévitch questionnait déjà: «L'altération dont l'autre est l'origine n'est-elle pas en toutes choses la première complication ?24 $^{12}$.

Dans le spectacle intitulé $60^{\text {ème }}$ parallèle de Philippe Manoury bâti sur un livret de Michel Deutsch, l'image du lieu public est introvertie (la scène montre une salle de transit d'aéroport à trois heures du matin). Ici, les sons de synthèse ne correspondent pas harmoniquement à ce que joue l'ensemble instrumental, ils sont par contre élaborés pour revêtir la couleur des voyelles qui sont chantées en direct sur scène. «Les tensions ne viennent pas d'une progression logique mais plutôt d'une éruption soudaine, comme c'est souvent le cas dans la réalité. Comme dans la vie réelle également, les phrases prononcées servent moins à expliquer cette réalité, qu'à les masquer. Elles n'en sont, dans leur apparente simplicité, que la partie visible d'un iceberg. C'est ainsi que les voix sont comme perdues dans la tempête, elles émergent de la musique de l'orchestre ou de l'ordinateur ${ }^{125}$ », explique le compositeur, pensionnaire régulier de l'IRCAM à Paris.

119 La guitare électrique est également présente dans La Tempesta (1977), L'Hommage à Charles Nègre (1986), L'Ile sonnante (1990), La Cité des saules (1997) de Hugues Dufourt (Cf. Castanet 1995, p. 162-164). Se référant à la partition de Saturne, Martin Laliberté a dénombré huit facteurs de construction des timbres dans le pupitre des guitares électriques, allant du spectre naturel à l'espace réverbéré (Cf. Laliberté 1995, p. 91). Lire également de Decleire 2011.

${ }^{120}$ Dans cette pièce, Alain Bancquart-alors membre du CRISS-a inséré deux guitares électriques qui, munies de pédale de double octave basse, ont pu remplacer avantageusement le jeu des contrebasses traditionnelles ( $c f$. Castanet 1995, p. 326).

${ }^{121}$ Dans le domaine de la mixité, signalons aussi Guitar Trio (1977) de ce guitariste nord-américain, partition qui combine allègrement les effets de la musique minimaliste (boucle répétitive, monodique sur la note Mi) avec la rythmique carrée du Punk Rock (avec pulse additive de batterie).

${ }^{122}$ Cette pièce hors norme est écrite pour cent guitares électriques, guitare basse et percussion.

123 Cf. Castanet 2003, p. 101-102.

124Jankélévitch 1960, p. 110.

125 Cf. Pour plus de renseignements, consulter : Manoury, Deutsch, Strosser 1998, p. 4-7. À lire aussi : Manoury 1998, p. 211-212. 
Voici reproduit un extrait du livret de Michel Deutsch qui débouche subrepticement sur une musique de Rock mettant notamment en valeur un solo déchirant de guitare électrique au travers d'un instant aux allures véristes (car pour accuser le ressort dramaturgique, un poste de radio doit mettre en lumière les reliefs hurleurs d'une musique pop).

Haut-parleur : «... Nous prions instamment le propriétaire du sac de voyage de couleur bleue qui se trouve sur le chariot dans le hall de transit de bien vouloir se présenter à la porte sept» (Les gens qui dormaient se redressent, se frottent les yeux, s'interrogent en s'étirant, interrogent leurs voisins).

La Femme : Mon Dieu! Mon Dieu !

L'Homme au transistor : Vous avez peur?

La Femme : Un sac abandonné !

L'Homme au transistor : On va tous sauter !

La Femme (tout le monde) : Mais il faut faire quelque chose!

L'Homme au transistor : Ne bougez pas ! C'est une sorte de mise à l'épreuve ! Dites-vous bien que c'est une mise à l'épreuve.

La Femme : Il faut... Il faut faire quelque chose !

L'Homme au transistor : Il n'y a rien à faire, madame. Attendre. C'est tout ce qu'il y a à faire. Tenez, je vais vous faire écouter quelque chose (Il allume le transistor. On entend un rock'n roll qui, peu à peu, va devenir assourdissant).

La Femme : Je vous en prie, s'il vous plait ! (L'Homme au transistor augmente le son)

La Femme : Arrêtez cette musique !

L'Homme au transistor : La nuit est belle.

Un passager : Arrêtez ça ! Qu'est-ce que vous cherchez? Vous cherchez à effrayer cette dame? Mais qu'est-ce que vous cherchez, bon Dieu ! ...

L'Homme au transistor : À votre avis, qu'est-ce que je cherche? (Il met le transistor à fond.... $)^{126}$

\section{LA GUITARE BASSE}

De Krzysztof Penderecki dans l'opéra Les Diables de Loudun (1969) à Benjamin de la Fuente dans Freewheel (2011) en passant par les opus de Mauricio Kagel, Leonard Bernstein, Louis Andriessen, Mark-Antony Turnage, Pelle Gudmundsen-Holmgreen, Michael Nyman, Fred Frith, René Lussier, Marc

${ }^{126} C f$. « Livret de l'opéra », CD 6oéme parallèle, Munich, Naxos nº 8.554249/50, 1998, p. 19. 
Monnet, Friedrich Zehm, Heiner Goebbels, Philippe Hurel, Kasper T. Toepliz ou Laurent Aglat... la basse électrique a royalement réussi à débrider le seuil du registre grave classique en complétant une instrumentation souvent pertinemment adaptée ${ }^{127}$. Benjamin de la Fuente a composé bon nombre de pièces alliant guitare électrique et basse électrique. Dans le corpus bigarré de ce violoniste improvisateur, citons par exemple Return/à la source (2010) ou Bypass (2011), deux partitions intégrant même la batterie à l'ensemble instrumental. De même, élève de Louis Andriessen en Hollande, Steve Martland a systématiquement inséré dans ses nomenclatures le couple guitare électrique/guitare basse (parfois avec corde grave de si bémol). En témoignent Remix (1986) pour dixtuor, Principia (1989) pour dixtuor, Dance Works (1993) pour nonetto, $M r$. Anderson's Pavane (1994) pour dixtuor, Horses of Instruction (1994-95) pour onze musiciens, Beat the Retreat (1995) pour onze musiciens, Rick (1996) pour onze musiciens, The Thistle of Scotland (1996) pour dixtuor, Eternal Delight (1997) pour onze musiciens, Terminal (1998) pour quintette ou sextuor... «La pop et le rock m'ont certainement inconsciemment influencé. Ce que j'aime dans ces musiques, c'est le côté physique, ce qui pousse à danser ${ }^{128}$ ", a admis le compositeur anglais.

En outre, composée de la guitare basse et de la batterie, la section rythmique de l'orchestre Rock se reconnait «monstrueusement» dans la partie terminale de Digital (2003) de Franck Bedrossian, partition composée pour contrebasse, percussion et électronique. «Le rock final fait office de résolution esthétique, manière de lever le voile pour annoncer une fin imminente [...] L'intensité sonore semble alors s'affranchir du seuil d'audibilitén ${ }^{129}$ ", relève Omer Corlaix. Jouant à dessein sur «l'excès de son ${ }^{130}$ » et sur le potentiel insoupçonné d'une «bruitologie performantielle ${ }^{131}$ », Franck Bedrossian ${ }^{132}$ a avoué à Bastien Gallet en 2007 avoir été ébloui à la fois par le «son saturé, sale, distordu» des musiques du groupe Velvet Underground et par les textures singulières développées notamment par les Stooges (sans parler des références à la Noise japonaise) ${ }^{133}$. De ce fait, en 2009, à Donaueschingen, Harry Halbreich a rangé ce compositeur parmi les membres de la génération des «nouveaux brutalistes»: "Maniant avec un maximum de violence tant une rythmique subjuguante (sic) qu'une amplification sonore visant expressément à la saturation et à la distorsion des fréquences, ils abolissent la frontière entre "son" et "bruit"134», a remarqué tout de go le musicologue belge.

127 Castanet 1998, p. 1112.

${ }^{128}$ Cf. Lelong 1996, p. 258.

129 Corlaix 2008, p. 6.

${ }^{130}$ Cf. Bedrossian 2008, p. 15-20.

${ }^{131}$ Cf. Castanet 2010, "Pour une bruitologie performantielle».

132 Auteur entre autres de partitions comme Charleston (2005-07) pour ensemble ou comme Bossa Nova (2008) pour accordéon.

133 Bedrossian 2008, p. 21, p. 22 et p. 42. Sur les mouvements post-rock, prière de lire : Robert 2011.

134 Halbreich 2009, p. 10. 


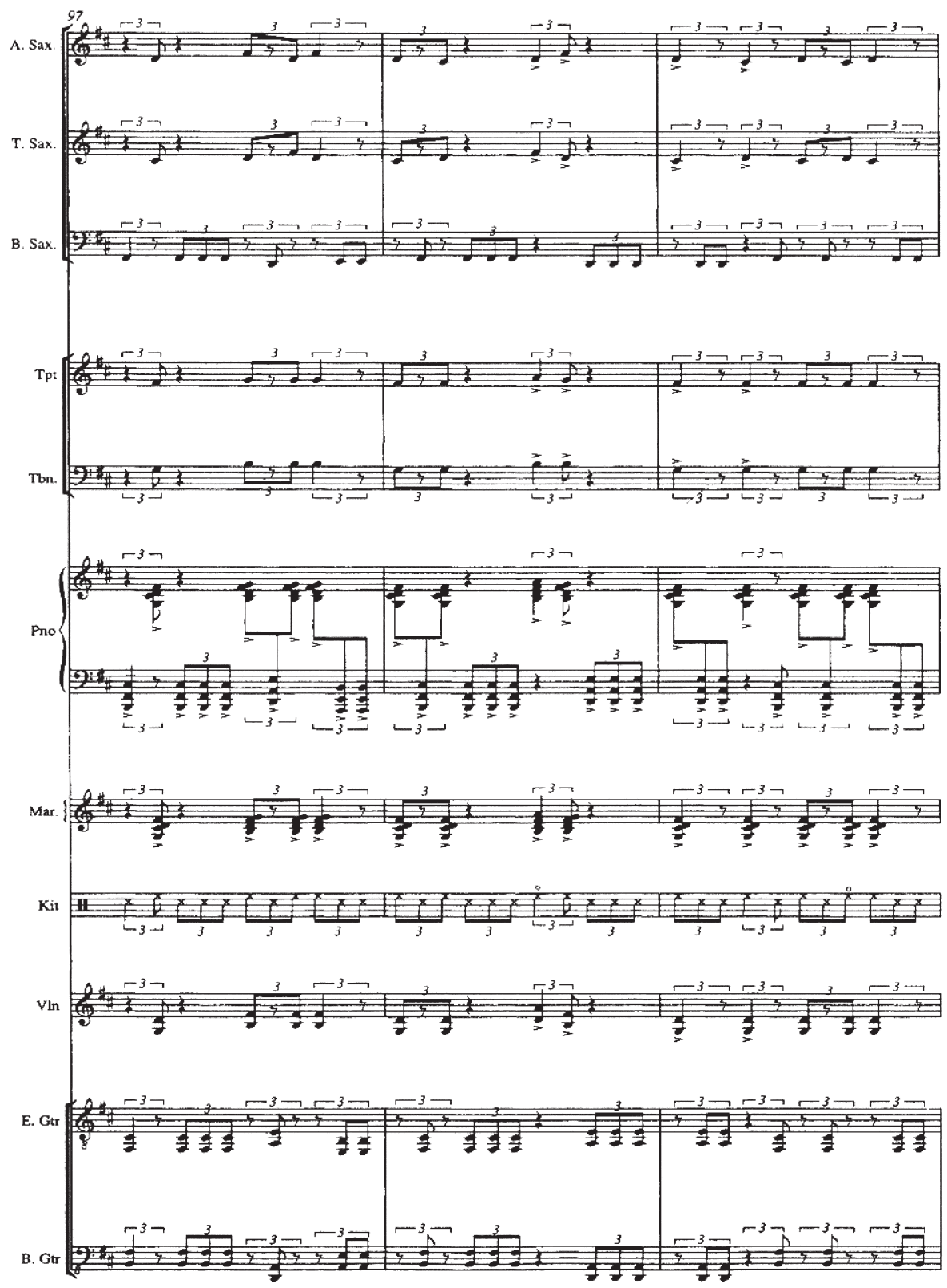

Exemple C: Mesures 97-99 extraites de Horses of Instruction de Steve Martland. Jeu de doubles cordes en homorythmie pour les guitare électrique et guitare basse. (Mainz, Éditions Schott, ED 12482, 1995, p. 18) 


\section{LA BATTERIE}

Présente autant dans les instrumentations de Luciano Berio (Laborintus 2-1963-65) que d'Alfred Schnittke (Première Symphonie - 1969-72), de Léonard Bernstein (Mass - 1971) que de Christopher Rouse (Bonham - 1988), de Michael Daugherty (Jackie O - 1993 - sur un livret de Wayne Koestenbaum) que de John Adams (I Was Looking at the Ceiling and Then I Saw the Sky - 1995 - sur un livret de June Jordan) ${ }^{135}$, de Brian Wilshere (Metropolis 1996-ou Zamalamaz - 2006) que de Jean-Philippe Goude (Rock de chambre - 2001 - pour ensemble avec violes de gambe et batterie) ou autant dans les opus d'Éric Fischer (Billet Doux - 1997) que d'Olivier Le Goas (Passages 200o), autant dans les combinaisons instrumentales de Pierre Tangay (Quatuor de batteries - 2001) que de Sébastien Gaxie ${ }^{136}$ (Live Sampling 1 - 2011) ou de Benjamin de la Fuente (Les Bretteurs - 1995, Manège - 2001, Flip - 2011), la batterie entremêle d'une manière complémentaire les sonorités typiques de peaux et de métaux frappés par les mains et par les pieds.

De Michael Finnissy (Ru Tchou - 1975 - pour batterie) à Theo Loevendie (New York - 2003 - pour percussion solo), les instruments basiques de percussions - caisse claire, toms aigu, medium, basse, grosse caisse, cymbales (crash, ride, rockride), cymbale charleston (Hi-hat) - ont, entre autres, servi à merveille le cliché binaire de la rythmique en accentuant la scansion bornée demandée par le Rock. Pierre Jodlowski, qui a composé passablement pour la percussion, nous a dit avoir toujours intégré ce pupitre (présentant la plupart du temps des hauteurs non tempérées) dans son travail en y profilant «un point de jonction» naturellement issu des mondes du rock et du jazz ${ }^{137}$. De ce compositeur, mentionnons juste au passage les différentes versions (avec vidéo ou pas) de Time \& Money (2005), partition qui, au demeurant, ne cache pas son apparentement à l'âge d'or des Pink Floyd. Citons également Bonham (1988) de Christopher Rouse écrit pour huit percussionnistes. Pensée comme une ode aux batteurs de rock en général, et au drummer de Led Zeppelin en particulier, cette partition cite à dessein des passages congrus de When the Levee Breaks ou de Custaud Pie and Royal Orleans.

Conçu pour un batteur, Tire-Ti.Ke-Dha (1979) de James Dillon met en jeu des descentes de toms typiques et en espace des lieux communs rythmiques issus du Rock and roll. En l'occurrence, les divers gestes percussifs côtoient roulements sur peau, rim shots et tintinnabuli de cloches. Si le musicien

135 Outre des guitares électriques, saxophone et synthétiseurs, l'orchestre de ce " songplay " utilise une batterie Rock et des percussions MIDI. Détachée de tout sentiment avant-gardiste, cette comédie musicale en chansons fait entendre un langage pop non dénaturé empruntant tantôt au Jazz, tantôt au Blues, tantôt au Gospel, tantôt au Rock...Néanmoins, Renaud Machart juge que «I Was Looking at the Ceiling and Then I Saw the Sky est trop raffinée pour être un musical dans l'esprit du temps et trop populaire pour séduire les fractions les plus sévères de la musique contemporaine " (Machart 2001, p. 123). Extrait de la musique du film The Glare, "City of Turin » de Michael Nyman atteindra sans scrupule la même forme de pastiche d'aura simpliste.

136 Élève de Brian Ferneyhough et d'Emmanuel Nunes (entre autres), Sébastien Gaxie est l'auteur de trois pièces pour big band de Jazz écrites en 1988 : Die Lüge, Lomir zich iberbeten et Undzer shtetel brennt, ainsi que d'un opus électroacoustique intitulé Cyber Punk (2006).

137 Cf. Castanet 2007, p. 39 
britannique fait explicitement référence à la musique populaire (tant par l'instrumentarium - une batterie jazz-rock étendue - que par la panoplie d'idiomes convoqués), il met néanmoins un point d'honneur à créer un contexte de «vitalisme ${ }^{138}$ ». En fait, la règle du jeu semble consister à s'échapper le plus souvent d'une rythmique carrée basique, contrairement à Traumbilder ou à Labyrinth de Fritz Hauser écrits eux aussi pour éléments de batterie rock mais qui ressassent sans aucune fantaisie les clichés primaires légués par la musique pop ${ }^{139}$. Dans la même veine, le compositeur néerlandais Theo Loevendie ${ }^{140}$ a désiré accuser sans scrupule des éléments «rocky» (voire «funky») dans sa partition pour batterie intitulée New York ${ }^{141}$. Entre différence et répétition, il demande même au percussionniste d'improviser plus longtemps (ad libitum) au sein des diverses carrures présentées s'il parvient stylistiquement au «right feel».

Dans l'exemple qui suit (compulsez les mesures 19 à 24 jouées à un tempo métronomique de 112 à la noire), l'amateur éclairé peut remarquer l'alternance de deux coups de grosse caisse à pédale accentués à la manière des rockers (tantôt en noires, tantôt en croches - voir la partie écrite sous la première ligne de la portée) ainsi que les quatre doubles croches notées sur le deuxième temps entre autres ${ }^{142}-$ jouées sur les toms et qui sont réitérées d'une mesure sur l'autre (sauf à la mesure 22).

Dans Trois Miniatures en Rock de Chambre (1999-200o) d'Éric Fischer ${ }^{143}$, support électronique et tutti instrumental acoustique accompagnent nerveusement la pulsation binaire d'un rock «savant». Dans le troisième mouvement, la rythmique principale est soutenue exclusivement par la scansion typée des drums $^{144}$. Chacun sait que le rapport au timbre - parfois connoté ou démesuré à outrance, simplement amplifié ou dénaturé volontairement - est devenu l'axe paramétrique moteur de la modernité du second vingtième siècle. Dans ce cadre, se penchant sur les expressions sonores des années 1990, Célestin Deliège n'a-t-il pas observé que le timbre s'est inscrit «au sein de la modernité musicale comme le facteur le plus apte à la féconder ${ }^{145}$ »? À l'image d'opus révélateurs de Josef-Anton Riedl (Paper Music), de Pierre Boulez (Dialogues de l'ombre double) ou de Jonathan Harvey (Tombeau d'Olivier Messiaen), la pièce $2 \times 5$ (2009) de Steve Reich a montré l'énergie débridée (ici assumée dans

138 « Au sens large de vitalité intense dans l'œuvre musicale (non en tant que doctrine) », note Darbon 2008, p. 223.

139 Cf. CD Solodrumming 1999.

${ }^{140}$ Auteur d'opéras de chambre et de musiques de scène, ayant à son catalogue bon nombre de partitions de musique de chambre, Theo Loevendie (né en 1930 à Amsterdam) est par ailleurs un jazzman renommé.

${ }^{141}$ Morceau inaugurant le premier volume des Recital Pieces pour percussion solo de Theo Loevendie.

${ }^{142}$ Comparez également les troisième et quatrième temps de ces mesures, ils sont identiques pour les mesures 19,20 et 23 .

143 Saxophoniste, Éric Fisher a été fondateur du collectif rock Adélaïde (1976-200o).

${ }^{144 C}$ f. Éric Fischer 2002.

145 Deliège 1993, p. 42. 

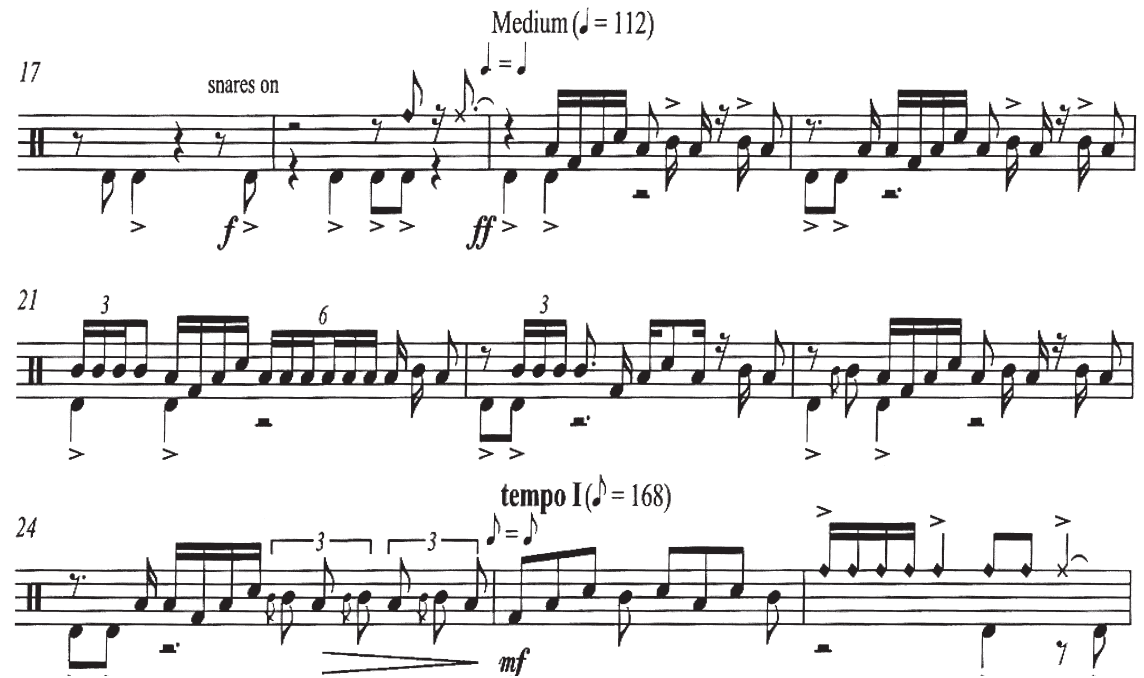

Exemple D: New York pour batterie de Theo Loevendie (Hamburg, Éditions Peermusic, 2003, p. 2)

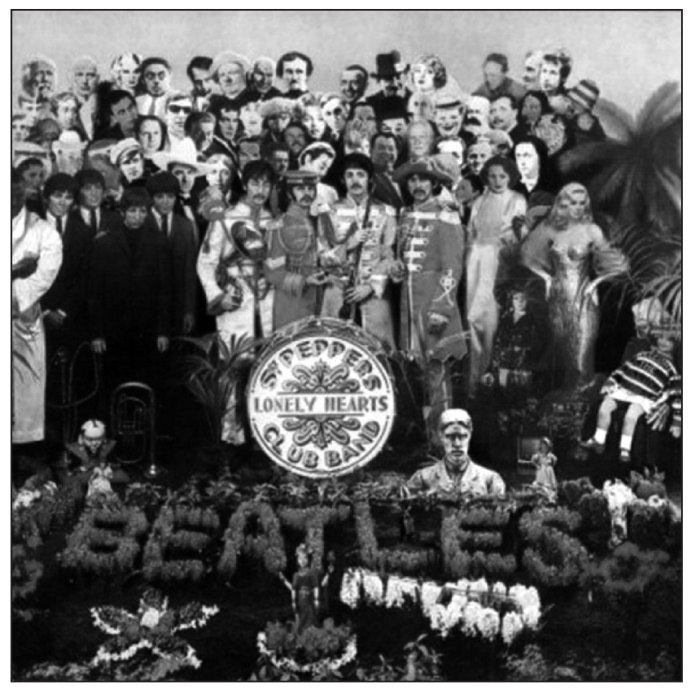

Figure 2. Pochette du disque 33 tours des Beatles intitulé Sgt Pepper's Lonely Hearts Club Band (1967)

un caractère pleinement «rock») d'un quintette amplifié se confrontant à des doubles fantomatiques enregistrés au préalable ${ }^{146}$.

${ }^{146}$ À noter également au niveau du reflet électroacoustique dû à la bande magnétique répliquant le matériau de la source primaire, citons les divers arrangements des Counterpoints de Steve Reich réalisés en 2010 par la percussionniste Kunito Kato. 
Figurant sur la photographie de groupe de la pochette discographique de Sgt Pepper's Lonely Hearts Club Band (1967) ${ }^{147}$ des Beatles, Karlheinz Stockhausen (le $5^{\text {ème }}$ en haut à gauche) a démontré entre autres qu'on pouvait utiliser la «force d'illumination» timbrique de manière hédoniste: "pour jouir plus ou moins du son, comme dans le rock»: Ainsi, "on écoute le timbre, le son pour lui-même. Mais deux ans plus tard, c'est dépassé, parce que le monde a changé, l'intérêt s'est émoussé: le son n'est plus si neuf. Et il n'a rien fait découvrir de nouveau, à l'intérieur d'une composition donnée. Il n'y avait pas cette interaction entre le matériau et la forme $\mathrm{e}^{148}$ \%.

\section{MATÉRIAUX ET FORMES AU SERVICE D'UN ART HYBRIDÉ}

À l'aube des seventies, accompagné d'un orchestre Rock (guitares électriques, guitare basse, batterie), le Credo de la Messe (1971) de Leonard Bernstein a fait symboliquement référence à un perpetuum mobile intentionnellement kaléidoscopique. Touchant néanmoins fondamentalement à l'idée de sacrilège, cette musique qui se voulait à l'origine religieuse est restée pour certains totalement subversive. Faisant se côtoyer blues, rock, gospel, jazz, marche militaire, chant grégorien, fanfare tonale ou thème dodécaphonique, «le compositeur n'homogénéise pas les différents niveaux de style, sans toutefois les distinguer avec soin. La façon dont ils sont mis en relation et entremêlés dépend de leur "place dans la vie". Car dans son hétérogénéité, Mass brosse, sous les espèces d'une œuvre d'art totale, le portrait de l'époque qui l'a suscitée ${ }^{149}$ », a noté Habakuk Traber.

Une génération après Saturne de Hugues Dufourt, une des synthèses les plus réussies du populaire avec le savant s'est reflétée au travers du talent énergisant de Fausto Romitelli (notamment grâce à En Trance - 1995-96, Professor Bad Trip - 1998-2000 - ainsi qu'à An Index of Metals - 2003). En effet, hormis l'instrumentarium intégrant ordinairement guitare électrique, basse électrique avec maints effets, mais aussi synthétiseurs, claviers-maîtres MIDI contrôlant échantillonneur et bande quadriphonique..., il est facile de repérer dans la partition de En Trance (construite en trois volets sur un texte extrait du Livre des Morts tibétain) de Romitelli, les divers critères d'organisation rythmique (notamment le jeu sec et musclé avec accents décalés) typiques de l'agencement répétitif des cellules fondamentales jouées par les rockers bassistes des années $1970^{150}$.

Enfin, dans le light show intitulé An Index of Metals, Fausto Romitelli n'a pas hésité à faire référence au genre collectif de la Rave party des nineties tout en agençant la visée «rituélique» de son entreprise vers les nuées de la «transe

147 Cet album qui est considéré par beaucoup comme le chef-d'œuvre du quatuor britannique sera qualifié de «meilleure œuvre rock de tous les temps » (cf. Leroy 2010, p. 14-17).

${ }^{148}$ Propos recueillis par Cott 1979, p. 28.

149 Traber 2004, p. 12.

150 Karlheinz Stockhausen disait qu'il n'était pas contre les formules répétitives dites " primitives » qui accompagnent les transes, dans le Rock (cf. Cott 1979, p. 28). 
lumino-sonore ${ }^{151}$ ». Dissertant sur le thème du musicien comme «virus», il remarquait que:

beaucoup plus que les écrivains, les cinéastes et les artistes, les compositeurs aujourd'hui sont obligés de se taire: parce que l'industrie culturelle empêche les personnes d'écouter et la normalisation des esprits supporte seulement la charge de produits pré-confectionnés très facilement digestibles. Certains d'entre nous s'enferment de plus en plus dans des langages privés et ésotériques; d’autres essaient fébrilement de reconquérir, sinon la faveur, du moins la tiède indulgence du public de la musique classique. D’autres cependant, acceptent le défi avec le monde, ou plutôt, avec le grand show médiatique et sponsorisé qui le remplace, et tentent de marcher sur ce même fil de laine qui sépare le banal de l'obscur, l'excès d'information de l'excès de redondance, l'impossibilité de communiquer de la facilité de transmettre des messages prédigérés ${ }^{152}$.

En fait, si les relations à l'autre et à la collectivité restent primordiales, les gestes imposés par le corps et la danse ne le sont pas moins. Dans ce cadre, influencé par les musiques rythmiques populaires, Steve Reich avouait sans fard: «Ma musique a du "beat" et peut-être peut-on danser dessus ; en ce sens, elle est plus proche du rock and roll que ne l'est la musique de Boulez, Stockhausen, Berio, Carter, Cage et Crumb. Je comprends cela ${ }^{153}$ ». D'autres seront ouverts à toutes propositions soniques et comportementales. Ainsi, parmi tant d'autres, Vittorio Montalti1 ${ }^{154}$ confessera qu'un compositeur «doit puiser dans le jazz, dans le rock, qui ont un autre rapport avec le corps. On ne peut pas danser sur Mozart! 155 »

Comme l'examine Mikel Dufrenne dans "Art et technologie», il arrive que l'entreprise de l'art révèle «la technologie à elle-même en subvertissant son usage, en séparant résolument sa vérité technique de sa vérité sociale ${ }^{156} \%$. Complémentaire à la recherche ethnologique de la couleur timbrique (grâce à la technologie de l'amplification, de la distorsion ou de l'hybridation électronique) ou au dépassement fatal (et néfaste) du seuil d'audibilité, l'appareil rythmique (assimilé au groove) ${ }^{157}$ tient également lieu d'étendard aux musiciens savants qui ont désiré, en un clin d'œil, faire référence à l'univers mouvementé et tonitruant du Rock ou de la Pop. «En cela, la Pop music/rock s'inscrit comme le prolongement logique du Rock'n roll: même aliénation par normes rythmiques - rythme(s) simple(s) et régulier(s), garant(s) d'une reconnaissance/consommation immédiate ${ }^{158} »$, relèvent de concert Philippe Daufouy et Jean-Pierre Sarton.

${ }^{151} C f$. Castanet, «La poétique musicale de Fausto Romitelli : de l'extravagans du matériau à l'ekstasis de la psyché » 2005, p. 89-114.

152 Romitelli 2001, p. 148-149.

153 Reich 1981, p. 162.

154 Compositeur italien né en 1984, Vittorio Montalti a été élève-entre autres-de Luca Francesconi, Ivan Fedele, Luis de Pablo et Toshio Hosokawa.

155 Montalti 2010, p. 102.

${ }_{156}$ Dufrenne 1981, p. 154.

157 Cf. Moore 2003, p. 838-839.

158 Daufouy et Sarton 1972, p. 172. 

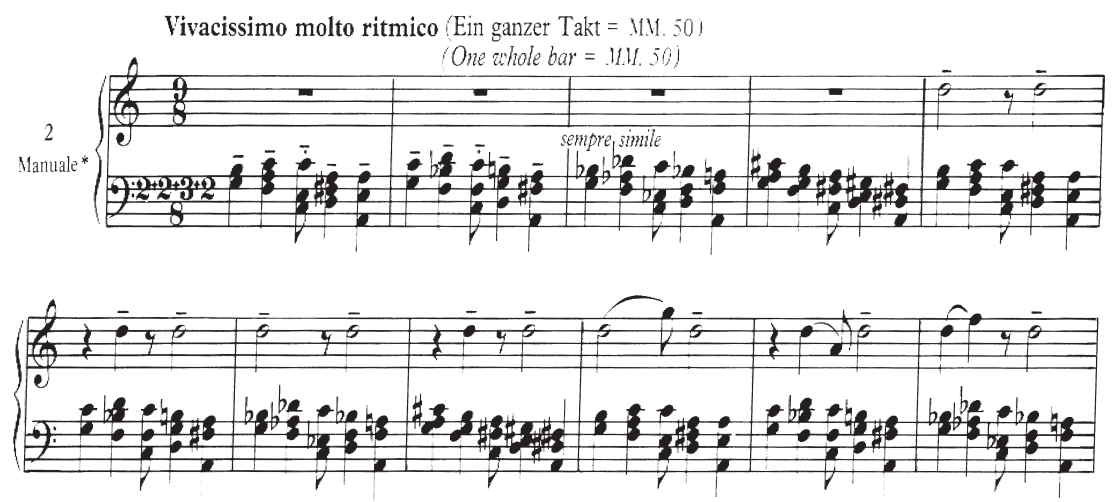

Exemple E: Début de Hungarian Rock pour clavecin de György Ligeti. (Mainz, Éditions Schott, 1979, p. 5)

Bon nombre de compositeurs de la génération née dans les années 1950 et après (Philippe Manoury, Pascal Dusapin, Dominique Lemaître, Denis Pousseur, Claude Ledoux, Andrea Liberovici ...) admettent qu'ils ont été sincèrement bercés par le phénomène du worldbeat et de la Global Pop ${ }^{159}$. En fait, l'histoire intéresse «celui qui a le goût de la conservation et de la vénération, celui qui se tourne avec amour et fidélité vers le monde d'où il vient et dans lequel il s'est formé ; par cette piété, il s'acquitte en quelque sorte de son existence ${ }^{160}$ ", pensait déjà Friedrich Nietzsche. Le compositeur Philippe Hurel énonce par exemple que «le rock et le jazz, plus que jamais nous ont permis de penser le rythme autrement et de sortir de l'éducation occidentale du rythme totalement obsolète et inefficace à mon sens ${ }^{161}$ ». Typiques par les impacts réguliers qui caractérisent leur répétitivité obsédante, les cellules percutées sur les temps (forts) de la mesure se reconnaissent alors autant dans Cobra (1984) de John Zorn que dans De Stijl (1984-85) de Louis Andriessen ${ }^{162}$. Néanmoins, remémorons-nous la question naïve posée par Yoko Ono à John Lennon (ex-membre des Beatles): "Mais pourquoi employez-vous ce beat tout le temps? Le même beat, pourquoi ne faites-vous pas quelque chose d'un peu plus compliqué ?163" Du point de vue de la musique «sérieuse», il est alors possible de montrer cette légère inflexion du complexe vers le plus simple.

159 Cf. Bohlman 2002.

160 Nietzsche 2000, p. 515 .

161 Hurel 2001, p. 32.

${ }^{162}$ Né en 1939, ce compositeur hollandais a déclaré : "C’est moi qui ai choisi d’être influencé par le jazz comme par les chansons de rock. Je vous rappelle que je suis un fan de Janet Jackson! " (cf. Lelong 1996, p. 39).

163 Cf. Rolling Stone, $\mathrm{n}^{\mathrm{o}} 74,1973$, p. 33. 
Ainsi, de l'Hungarian Rock (1978) pour clavecin ${ }^{164}$ de György Ligeti (inspiré par une chanson des Beatles) ${ }^{165}$ au Rock de chambre (1999-200o) d'Éric Fisher, en passant par exemple par Principia (1989) de Steve Martland, carrures régulières, ostinati rythmiques et schémas harmoniques désirent montrer une volonté commune de cristalliser un discours à la connotation populaire évidente. Pour élaborer la partition d'Hungarian Rock, György Ligeti a ironiquement tressé les racines hongroises de son pays natal avec les banderoles bariolées de la pop music européenne. Dans les faits, para folklorique et faussement classique ${ }^{166}$, la mélodie clavecinistique (figurée à la main droite) se fonde sur une succession d'accords kaléidoscopiques, en ostinato, mus par une carrure de quatre mesures. Quant à la ligne de basse - identique à chaque mesure: cinq valeurs de notes ( $s o l, f a, d o, r e ́, l a)$ agencées en un $9 / 8$ de $2+2+$ $3+2$ croches -, elle est harmonisée différemment dans chacune des présentations. Mais, «à l'audition, conclut Pierre Michel, Hungarian Rock fait immédiatement penser à certaines musiques populaires (jazz, rock), surtout par son style très rythmique ${ }^{167}$ ».

\section{ÉLÉMENTS CONCLUSIFS}

Comme le soulignait Theodor W. Adorno, «la question des critères musicaux se trouve prise dans la tension d'une alternative aussi problématique que stérile ${ }^{168} »$. Ainsi, regorgeant de puissance sonore paroxystique et de vitalité spatio-dynamique inouie, l'art hybride issu de la présence de la Pop Music auréolée à la fois de «praxis» et de «mythologie ${ }^{169}$ » - mêle souvent simplicité naïve et complexité manifeste. Parfois métaphorique, parfois réelle, cette volonté d'union du Rock en tant qu' «art mineur ${ }^{170}$ » ou «inférieur ${ }^{171}$ » et de la Musique contemporaine en tant qu'«art majeur» ou «supérieur» a toujours

${ }^{164}$ Polyvalent au XXe siècle, cet instrument à clavier est présent (acoustiquement ou synthétiquement) autant dans Métamorphose de Bernd Alois Zimmerman que dans les arrangements de songs des Beatles par Louis Andriessen pour les récitals de Cathy Berberian, autant dans Sinfonia de Luciano Berio que dans les chansons de Michel Polnareff des années 1960-70, autant dans Jakob Lenz de Wolfgang Rihm que dans le catalogue de François-Bernard Mâche (instrument amplifié ou non), autant dans les Études transcendantales de Brian Ferneyhough que dan Birl de James Dillon, autant dans les Carillons pour les heures du jour et de la nuit de Maurice Ohana que dans les musiques de film de Michael Nyman, autant dans Der Pflaumenbaum de Heiner Goebbels que dans Polaris de Stratovarius, autant dans les musiques du groupe anglais Nice que dans One Way Bedroom (2003) du Hollandais Mayke Nas où-selon lui-l'instrument est traité « comme une machine à écrire »...

${ }_{165}$ Cf. Michel 1995, p. 106.

${ }^{166} \mathrm{La}$ partition qui porte le sous-titre de "chaconne » attribue des « mordants " baroques à certaines noires ou croches (voir les mesures $30,32,38$, par exemple).

167 Michel 1995, p. 106.

168 Adorno 2006, p. 142.

169 « Consommée esthétiquement, la culture de masse développe, au-delà de l’esthétique, une praxis et une mythologie. C'est-à-dire qu'elle déborde l'esthétique aussi bien vers le réel que vers l'imaginaire », écrit Edgar Morin (Morin 1962, p. 125). Consulter également : Hennion 1991.

170 Mazzoleni 2011, p. 7.

${ }^{171}$ Leonard Bernstein s'exclamait : « Hélas! De nombreuses personnes considèrent que la musique populaire est inférieure. $\mathrm{Si}$, par musique populaire, on entend coûte que coûte une musique inférieure, je ne suis pas du tout d'accord : une symphonie n'est pas meilleure qu'une bonne chanson simplement parce que c'est une symphonie !» (Bernstein 1993, p. 62). 
alimenté des jugements souvent hâtifs. En 1936, l'ethnologue Marcel Griaule écrivait avec conscience que les hommes «devraient savoir que chaque culture a sa valeur et qu'il est vraiment par trop imbécile de décerner à la sienne propre le premier d'excellence ${ }^{172}$ ».

En fait, cette association a encouragé les troubles de la transgression et de l'utopie comme les plaisirs de la salissure et de l'hybridation - «singulier et détonant mélange, mais quoi de moins surprenant au fond si l'on observe l'évolution de la rock music depuis 1954 ? ${ }^{173}$ ", tient à rendre compte le sociologue Paul Yonnet au centre de son essai sur Jeux, modes et masses. Au début des années 1960-époque où le parfum du Rock embaumait avec singularité les boutiques des disquaires et les juke boxes des bistrots - on remarquait déjà ceci: «il n'existe pas d'absence de culture, il n'existe qu'une opposition à la culture $^{174}$ » (Franco Fabbri a même évoqué la présence organisationnelle du Rock In Opposition) ${ }^{175}$. Projetant les traits d'une «sonodoulie ${ }^{176}$ » décomplexée, cette objection n'a pu être vaincue ou régulée que par l'arrivée de l'esprit somme toute postmoderne - de "globalisation ${ }^{177}$ » qui a caractérisé le passage au XXI ${ }^{\mathrm{e}}$ siècle. Ainsi, l'ampleur de la vague populiste ou démagogique renversant sans honte les valeurs élitistes a conforté l'océan des dilutions et des dissolutions. "La disparition de la distinction entre art "noble" et art "populaire" se manifeste dans toutes les sphères de la culture, puisque l'on voit aussi bien Pavarotti chevaucher les sommets du hit-parade que des "punks" interpréter de la musique classique ${ }^{178}$ ", remarque encore Fiona MacLean.

Dénonçant de faux consensus, Serge Chaumier a alors montré dans son livre intitulé L'Inculture pour tous ${ }^{179}$ que la culture souffrait d'un écartèlement circonstancié ou d'un éclatement des contextures nobles, vulgaires, ésotériques ou intellectuels présidant à chaque expression artistique librement consentie. Dans ces conditions plus proches de la rupture que de la soudure, en tant qu'expression criarde de la société mondiale, la World Music a ainsi pâti de son mode avilissant de banalisation et de neutralisation identitaires ${ }^{180}$ après avoir fait figure de «révélateur de mutations sociales et culturelles essentielles ${ }^{181}$ ». Certes - nous l'avons déjà mentionné - parmi maints exemples au regard de concordances sociopolitiques ou socioculturelles, le Rock n'est ni plus ni moins qu'un maillon de la chaîne de l'expression de la vie. «L'art vécu devient plus qu'un symbole - explique alors Henry Torgue: la musique est une fin en soi, qui porte en elle la critique et le rêve, l'appel à la révolte et le frein à la révolution,

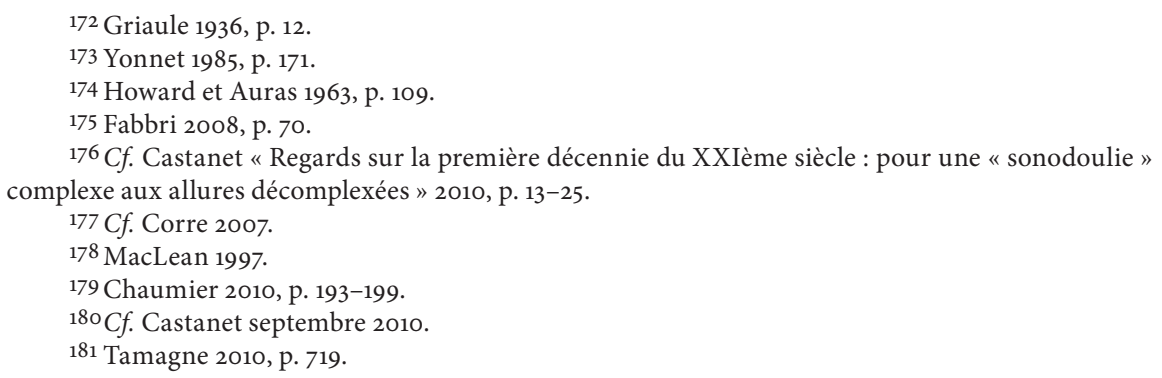


la chaleur des rituels collectifs et l'énergie brûlante du présent ${ }^{182}$ ». À l'opposé, pour d'autres esprits, «la culture rock est une véritable course vers le glaireux, le nauséeux, l'excrémentiel où la bêtise camouflée sous la vulgarité est justifiée par l'insolence: une trouvaille, un coup de génie, le signe d'une époque ${ }^{183}$ ». (Rappelons pour la petite histoire que "nausée» et «bruit» ont en commun ce germe étymologique médiéval issu du vieux provençal «noyse $)^{184}$.

In fine, nous pourrions dire qu'à l'instar des signes mystificateurs qui ont transformé «la culture petite-bourgeoise en nature universelle ${ }^{185}$ », le Rock est devenu facteur idéologique de mythologie quotidienne. Ainsi, François Jouffa pense que «ce qui est fabuleux avec le rock'n'roll, c'est qu'il touche aujourd'hui aussi bien la mère de famille ou le sexagénaire nostalgique que le jeune loubard des périphéries urbaines, toutes confessions confondues ${ }^{186} \%$. À l'aube de ce nouveau millénaire, à écouter les échos routiniers de notre environnement urbain, sans oublier les cœurs mélancoliques comme les consciences libérées, la condition sociétale montre effectivement que le médium du Rock questionne encore quelques états d'âme illuminés. Alors que les tags et les graffiti populaires sont aujourd'hui exposés dans les plus grands musées du monde, les divers stimuli du Rock - à différents stades de leur stratification - ont été récupérés pour aboutir à de réelles partitions contemporaines, cette fois d'obédience savante.

«Le rock and roll ne mourra qu'avec la fin de l'humanité dont il représente pour beaucoup d'entre nous la création la plus belle et la plus achevée ${ }^{187}$ ", rêve encore Michel Rose.

[Très enrichi et complété, cet article émane d'une conférence prononcée à l'Université de Dijon, le 11 mars 2009, dans le cadre d'un Séminaire sur le Rock expérimental.]

\section{RÉFÉRENCES}

Adorno, Theodor W. 2006. Figures sonores, Écrits musicaux I. Genève : Contrechamps.

—.Mai 1971. «Sociologie de la musique», Musique en jeu nº 2. Paris : Seuil. 1982. Quasi una fantasia. Paris: Gallimard.

Alla, Thierry. 2008. Tristan Murail - La Couleur sonore. Paris : Michel de Maule.

Bacon, Tony. 2004. Le Grand livre de la guitare. Genève : Minerva.

- 1981. Rock Hardware: The Instrument and Technology of Rock. Londres: Blandford Press.

Barsamian, Jacques et François Jouffa. 2005. «Les différentes vies du rock», Histoire du Rock. Paris : Taillandier.

Barthes, Roland. 1957. Mythologies. Paris : Seuil.

Baudrillard, Jean. 1970. La Société de consommation. Paris : Denoël.

182 Torgue 1997, p. 110.

183 Georges Suffert, Le Figaro Magazine, 1988 (propos cités par Duhamel 1999, p. 465).

184 Cf. Castanet 1999 (rééd. 2007), p. 289.

185 Barthes 1957, p. 7.

${ }^{186}$ Barsamian et Jouffa 2005, p. 19.

187 Rose 1981, p. 188. 
1968. Le Système des objets. Paris : Denoël/Gonthier,

Bedrossian, Franck. 2008. «La monstruosité, de l'œil à l'oreille», De l'excès de son, coll. À la ligne. Champigny : 2e2m.

Bell, Daniel. 1976. The Cultural Contradictions of Capitalism. New York : Basic Book.

Béranger, Sébastien. 2006. «Des traditions permissives, ou la réappropriation des langages", Nunc $\mathrm{n}^{\circ}$ 14. Clichy : Cordevour.

Berio, Luciano. Mai 1971. "Commentaires au rock», Musique en jeu no 2. Paris : Seuil. (pour la version en langue française).

Bernstein, Leonard. 1993. Le Partage de la musique. Paris : Belfond.

Bizot, Jean-François. Mai 1971. "Les pop heureux ont une histoire», Musique en jeu, $\mathrm{n}^{0} 2: 77$. Paris : Seuil.

Bloch, H. et A. Niederhoffer. 1984. La Génération éclatée : loisirs et communication des adolescents, Bruxelles, sous la dir. de Roselyne Bouillin-Dartevelle. Bruxelles : Éditions de l'Université de Bruxelles.

Bohlman, Philip V. 2002. World Music. A Very Short Introduction. New York: Oxford University Press Inc.

Boulez, Pierre. 9 mars 2001. "Boulez tra avventura e tradizione», Il Manifesto. Bourdieu, Pierre. 1980. Le Sens pratique. Paris : Minuit.

Brackett, David. 2002. "Where's It Art?'-Postmodern Theory and the Contemporary Musical Field», Postmodern Music Postmodern Thought, sous la dir. de Judy Lochhead et Joseph Auner. New York : Routledge.

Castanet, Pierre Albert. 2010. "Pour une bruitologie performantielle», Le Performantiel noise, sous la dir. de Sébastien Biset. Bruxelles : revue (SIC)

- 2010. "Regards sur la première décennie du XXI" siècle : pour une "sonodoulie» complexe aux allures décomplexées», Composer au XXI siècle, Pratiques, philosophies, langages et analyses, sous la dir. de Sophie Stévance. Paris : Vrin.

Septembre 2010. «De la compromission identitaire de la musique contemporaine à l'époque postmoderne», Traditions, Créations, Instruments, Signes, revue Sonorités, $\mathrm{n}^{\circ}$ 5. Nîmes : Champ Social Éditions.

. 2010. "Les grimoires de Mnémosyne : pour un palimpseste des origines", Back to the Future - Rainy Days. Luxembourg : Philharmonie Luxembourg.

. 2010. "Ouvrir son cœur à la création» : notes sur l'art du ready-made sonore», Manières de faire des sons, sous la dir. d'Antonia Soulez et Horacio Vaggione. Paris : L'Harmattan.

. 2008. Quand le sonore cherche noise - Pour une philosophie du bruit. Paris : Michel de Maule.

. 2008. "Tristan Murail, l’artificier de la métamorphose», préface du livre de Thierry Alla, Tristan Murail - La Couleur sonore, IV. Paris : Michel de Maule.

. 2007. "Les champs de la percussion au XXème siècle», Le Geste et l'Esprit. Paris : Tschann Libraire. (coffret : livre bilingue, CD, DVD).

—. Novembre-décembre 2005. «Du Rap dans la musique contemporaine savante», L'Éducation musicale $\mathrm{n}^{0}$ 527-528:29-31. 
-2005. «La poétique musicale de Fausto Romitelli : de l'extravagans du matériau à l'ek-stasis de la psyché», Le Corps électrique - Voyage dans le son de Fausto Romitelli, sous la dir. d'Alessandro Arbo. Paris: L'Harmattan. _. 2003. «Opera Mundi - L'opéra pluraliste ou les vicissitudes socio-culturelles et socio-politiques de l'opéra contemporain», Composer un opéra aujourd'hui, sous la dir. de Béatrice Ramaut-Chevassus. Saint-Étienne : Publications de l'Université de Saint-Étienne.

1999 (rééd. 2007). «L'univers Pop et son ombre», Tout est bruit pour qui a peur - Pour une histoire sociale du son sale. Paris : Michel de Maule.

_. 1998 «Jazz, rock, rap savants», Histoire de la Musique, sous la dir. de Marie-Claire Beltrando-Patier, 1110-1116. Paris : Larousse.

1995. Hugues Dufourt -25 ans de musique contemporaine. Paris : Michel de Maule.

Caunes de, Antoine. 2010. Dictionnaire amoureux du Rock. Paris : Plon.

Caux, Daniel. 2009. Le Silence, les couleurs du prisme \& la mécanique du temps qui passe. Paris : Éditions de l'éclat.

Certeau de, Michel. 1993. «La culture dans la société», La Culture au pluriel. Paris : Seuil.

Ceserani, Remo.1997. Raccontare il postmoderno. Torino : Bollati Boringhieri editore.

Chastagner, Claude. 2011. De la culture rock. Paris : Presses Universitaires de France.

Chaumier, Serge. 2010. L'Inculture pour tous - La nouvelle utopie des politiques culturelles. Paris : L'Harmattan.

Cohn, Nik. 2004. Awophopalodsop Alophamboom - L'Age d'or du Rock. Paris : Allia.

Collins, Nick et Julio d'Escriván (dir.) 2007. The Cambridge Companion to Electronic Music. Cambridge : Cambridge University Press.

Cordes, Casper. 2006. «Musique et participation», Carnet de bord. Paris : $\mathrm{CDMC/Ensemble} \mathrm{Aleph.}$

Corlaix, Omer. 2008. «Le son, cet inconnu», livret du CD de Franck Bedrossian, Charleston. Paris : Sismal records.

Corre, Christian. 2007. «World Music : un objet virtuel? », Filigrane ${ }^{\circ}{ }^{5}, \mathrm{Mu}$ sique et globalisation.

Cott, Jonathan. 1979. Conversations avec Stockhausen. Paris : Lattès.

Darbon, Nicolas. 2008. Brian Ferneyhough et la Nouvelle Complexité. Notre Dame de Bliquetuit, Millénaire III,

-2007. Wolfgang Rihm et la Nouvelle Simplicité. Notre Dame de Bliquetuit : Millénaire III.

2006. Les Musiques du chaos. Paris : L'Harmattan.

Daufouy, Philippe et Jean-Pierre Sarton. 1972. Pop Music Rock. Paris : Champ libre.

Decleire, Vincent. Janvier 2011. «La guitare électrique de Jimi Hendrix à Hugues Dufourt», Études, Tome 414/1.

Deliège, Célestin. 1993. "L'écriture et ses mutations", Genesis no 4. Paris : ITEM/CNRS - Jean-Michel Place. 
Duchamp, Marcel. 1970. «Entretien avec Otto Hahn», VH 101, $\mathrm{n}^{\circ} 3: 116$.

Dufrenne, Mikel. 1981. Esthétique et Philosophie, tome 3. Paris : Klincksieck.

Duhamel, Jérôme. 1999. Le XXème siècle bête et méchant. Paris : Albin Michel.

Duteurtre, Benoit. 2006. Requiem pour une avant-garde. Paris : Les Belles Lettres.

Electric Body - Le corps en scène. 2002. Paris : Cité de la Musique / Beaux-Arts Magazine.

Fabbri, Franco. 2008. Il suono in cui viviamo - Saggi sulla popular music. Milan : Il Saggiatore.

Frith, Simon. 1978. Sociology of Rock. Londres : Constable.

Glissant, Édouard. 1997. Traité du Tout-Monde, Poétique IV. Paris : Gallimard. Griffiths, Paul. 1995. Modern Music and after. Oxford: Oxford University Press. Goetschel, Pascale. 2010. Histoire culturelle de la France au XX $X^{\text {ème }}$ siècle. Paris : La Documentation Française.

Gould, Glenn et Bruno Monsaingeon, 1985. Écrits II. Paris : Fayard.

Griaule, Marcel.1936. La Peau de l'ours. Paris : Gallimard.

Grignon, Jean-Claude et Claude Passeron.1989. Le Savant et le Populaire. Paris : Seuil.

Halbreich, Harry. 2009. «Cédric Dambrain - Une violence euphorique», Beyond - Essais et documents. Bruxelles : Ars Musica.

Hall, Stuart et Paul Du Gay (dir.) 1996. Questions of Cultural Identity. Londres": Sage.

Hennion, Antoine (dir.) 1991. Rock, de l'histoire au mythe. Paris : Anthropos.

Hoffmann, Raoul et Jean-Marie Leduc. 1978. Rock babies - 25 ans de pop music. Paris : Seuil.

Holmes, Thom. 2008. Electronic and Experimental Music. New York / Londres : Routledge.

Howard, Walter et Irmgard Auras. 1963. Musique et Culture. Paris : Presses Universitaires de France.

Hurel, Philippe. 2001. "Une musique résistante», Musiques actuelles, musique savante - Quelles interactions?, sous la dir. d'Éric Denut. Paris : L'Harmattan.

Jameson, Frederic. 1984.«Postmodernism, or the Cultural Logic of Late Capitalism ", New Left Review $\mathrm{n}^{0} 146$.

Jankélévitch, Vladimir. 1960. Le Pur et l'Impur. Paris : Champs Flammarion.

Kaltenecker, Martin. 2000. "François-Bernard Mâche», livret du CD Assai 222192 MU750.

Klein, Jean-Claude. Mars 1984. «Le syndrome de l'imitation», Show Biz, revue Autrement, $\mathrm{n}^{\mathrm{O}} 58$.

Kyrou, Ariel. 2002. Techno Rebelle. Paris : Denoël - X-trême.

Laliberté, Martin. Mars-juin 1995. "Orchestration, mixité et pianisme dans Saturne de H. Dufourt», Les Cahiers du CIREM no 35-36.

Lambotte, Marie-Claude. 2003. «Fonction du contexte dans le statut de l'objet esthétique», Effets de cadre - De la limite en art. Saint Denis : Presses Universitaires de Vincennes.

Lelong, Stéphane. 1996. Nouvelle musique. Paris : Balland. 
Lemery, Denis. mai 1971. "Musique contemporaine, pop-music et free-jazz, convergences et divergences", Musique en jeu, $\mathrm{n}^{0}$ 2. Paris : Seuil.

Leroy, Aymeric. 2010. Rock progressif. Marseille : Le mot et le reste.

Leterrier, Sophie-Anne. 2010. "Courants musicaux", Dictionnaire d'histoire culturelle de la France contemporaine, sous la dir. de Christian Delporte, Jean-Yves Mollier, Jean-François Sirinelli. Paris : Presses Universitaires de France.

Lewallen, Constance. Mai 1991. "Kunst ist eine Art Versuchslabor», DU-Heft, Composer John Cage, Zurich, Konzept wider den Zwang, ${ }^{0} 5$ : 66.

Ligeti, György. 2001. "Pensées rhapsodiques sur la musique en général et sur mes propres compositions en particulier», Neuf essais sur la musique. Genève : Contrechamps.

Louvier, Alain et Pierre Albert Castanet. 1997. L’Orchestre. Paris : Combre.

Machart, Renaud. 2001. John Adams. Arles : Actes Sud/Classica.

MacLean, Fiona. Janvier 1997. «Le passé est à vendre : réflexion sur le marketing des musées », Publics et musées, $\mathrm{n}^{\mathrm{O}}$ 11-12.

Mac-Luhan, Marshall. 1967. The Medium is the Message. New York-London : Bantam Books.

1968. Pour comprendre les médias. Paris : Seuil.

Maffesoli, Michel. 2008. "Raves (party)», Iconologies - Nos idol@tries postmodernes. Paris : Albin Michel.

Manoury, Philippe, Michel Deutsch, Pierre Strosser. 1998. «Naissance d'une œuvre commune», livret du CD $6 o^{e ́ m e}$ parallèle. Munich : Naxos $\mathrm{n}^{\circ}$ 8.554249/50,

Manoury, Philippe. 1998. «À n'importe quel moment — à propos de 6oéme parallèle», La Note et le Son, Écrits et entretiens, 1981-1998. Paris : L'Harmattan/L'Itinéraire.

Marengo, Renato, Michael Pergolano, Mario De Felicis. 2003. Enciclopedia del pop rock napoletano: Da Roberto Murolo ai Posse. Rome : RAI-ERI.

Marshall, Ingram. 2002. "A Sentimental Journey», livret du CD Naive and Sentimental Music, Nonesuch 7559-79636-2.

Masci, Franceso. 2011. Entertainment! Apologie de la domination. Paris: Allia. Mazzoleni, Florent.2011. L’Odyssée du Rock. Paris : Hors Collection.

Métivivier, Francis. 2011. Rock'n philo. Paris : Bréal.

Michel, Pierre.1995. György Ligeti. Paris : Minerve.

Moore, Allan F. 2003. "La musique pop ", Musiques. Une encyclopédie pour le $X X I^{e}$ siècle, "1. Musiques du $\mathrm{XX}^{\mathrm{e}}$ siècle», sous la dir. de Jean-Jacques Nattiez, 832-846. Arles-Paris : Actes Sud/Cité de la musique.

Morin, Edgar. 1994. Sociologie. Paris : Fayard.

1970. Journal de Californie. Paris : Seuil. 1962. L'Esprit du temps. Paris : Grasset.

Montalti, Vittorio. 2010. "Nu descendant un escalier», Carnet de bord. Paris : CDMC/Ensemble Aleph.

Murail, Tristan. 2001. "Le temps des convergences est révolu», Musiques actuelles, musique savante - Quelles interactions?, sous la dir. d'Éric Denut. Paris : L'Harmattan. 
Salabert.

Nietzsche, Friedrich. 2000. «De l'utilité et des inconvénients de l'histoire pour la vie», Considérations inactuelles, Euvres I, sous la dir. de Marc de Launay. Paris : Gallimard, coll. La Pléiade.

Ono, Yoko. 2004. "Voice Piece for Soprano», Pamplemousse. Paris : Textuel.

Pirenne, Christophe. 2005. Le Rock progressif anglais (1967-1977). Paris : Honoré Champion.

Plougastel, Yann 1997. Le Rock, Dictionnaire illustré. Paris : Larousse.

Quignard, Pascal. 1996. La Haine de la musique. Paris : Calmann-Lévy.

Quint, Laurent. 2010-2011. Rock'n'Roll - Les 100 premières fois. Paris : Gründ.

Ravet, Hyacinthe. 2010. "Les nouvelles frontières du métier de compositeur", Composer au XXI siècle, Pratiques, philosophies, langages et analyses, sous la dir. de Sophie Stévance, 27-40. Paris : Vrin.

Reich, Steve. 1981. «Entretien avec Cole Gagne», Écrits et entretiens sur la musique. Paris : Bourgois. (pour l'édition en langue française).

Ribac, François. 2004. L’Avaleur de Rock. Paris : La Dispute.

Richert, Francis. 2010. "Musiques actuelles amplifiées, vers une légitimité culturelle», Entre-deux n ${ }^{\circ}$ 10. Lyon : CFMI/Mômeludies Éditions.

Robert, Philippe. 2011. Post-punk, No wave, Indus \& Noise. Marseille : Le mot et le reste.

Rockwell, John. 1985. All American Music. London : Kahn \& Averill.

Rollin, Monique. 1994. "À propos du rock et de la musicologie», Le Rock - Aspects esthétiques, culturels et sociaux, sous la dir. d'Anne-Marie Gourdon. Paris : CNRS Éditions.

Romitelli, Fausto. 2001. "Il compositore come virus», Milano musica. Percorsi di musica d'oggi - Il pensiero e l'espressione. Aspetti del secondo Novecento musicale in Italia. Milan : Milano Musica.

Rougemont de, Denis. 1972. Penser avec les mains. Paris : Gallimard.

Rose, Michel. 1981. Pionniers du rock'n'roll. Paris : Albin Michel.

Rousselot, Philippe. 1995. "L'homme et l'instrument dans le répertoire libre», Les Cahiers de l'IRCAM, $\mathrm{n}^{\circ} 7$, Instruments. Paris : Ircam - Centre Georges Pompidou.

Roux, Alain. 1973. "La Musique pop» Musique et vie quotidienne - Essai de sociologie d'une nouvelle culture, sous la dir. de Paul Beaud et Alfred Willener. Paris : Maison Mame.

Rubin, Jerry. 1971. Do it. Paris : Seuil.

Russolo, Luigi. 1975. L'Art des bruits. Lausanne : L’Âge d'Homme.

Sabatier, Benoît. 2011. Culture jeune - L'épopée du rock. Paris : Fayard/Pluriel. Schneider, Michel. 1993. La Comédie de la culture. Paris : Seuil.

Shusterman, Richard. 1991. L'Art à l'état vif - La pensée pragmatiste et l'esthétique populaire. Paris : Les Éditions de Minuit.

Sibilla, Gianni. 2003. I linguaggi della musica pop. Milan : Strumenti Bompiani. Sontag, Suzan. 1966. Against Interpretation and Others Essays. New York : Dell. 
Soucy, Gaétan. Automne 1999. «Bâtons rompus sur la notion de culture, sur le rock'n'roll, sur la littérature jeunesse, sur Sid Vicious, sur toutes sortes d'affaires", Argument, Rock, Punk, Rap : culture ou imposture? II, $\mathrm{n}^{\mathrm{0}} 1: 29$. Stévance, Sophie. 2009. Duchamp, compositeur. Paris : L'Harmattan.

Tamagne, Florence. 2010. « Rock», Dictionnaire d'histoire culturelle de la France contemporaine, sous la dir. de Christian Delporte, Jean-Yves Mollier, JeanFrançois Sirinelli. Paris : Presses Universitaires de France.

Traber, Habakuk. 2004. "Une tranche d'histoire controversée», livret du coffret discographique HMC 901840.41, Mass de Leonard Bernstein. Arles : Harmonia mundi.

Torgue, Henry. 1997. La Pop-Music et les musiques Rock, Coll. Que sais-je? Paris: Presses Universitaires de France.

Tzortzis, Nicolas. 2010. «Amenable», Carnet de bord. Paris : CDMC/Ensemble Aleph.

Wakin, Daniel J. 2007. «From Lead Percussionist to Different Drummer», New York Times, 6 février.

Walker, John A. 1987. Cross-overs. Art Into Pop/Pop Into Art. Londres : Methuen. Yonnet, Paul. 1985. "Rock, pop, punk», Jeux, modes et masses - 1945-1985. Paris : Gallimard.

\section{Disques, Enregistrements}

Fischer, Éric. 2002. Même si la lumière... CD Daphénéo A 209.

Le Trésor de la langue.1989. AM 015 CD. Montréal : CAPAC.

MagnifiCathy - The many voices of Cathy Berberian (1928-1983). 1988. CD

Wergo WER 60054-50.

Rivet, Christian. 2009. 24 Ways upon the Bells. CD Naïve AM 183.

Solodrumming. 1999. Hat (now) CD ART 129.

We insist. 1960. Candid CCD79002.

\section{RÉSUMÉ}

Devenu depuis plus d'un demi-siècle facteur idéologique de mythologie quotidienne, le Rock a séduit bon nombre de compositeurs contemporains (John Adams, Luciano Berio, Steve Martland, Theo Loevendie...). Explorant cette problématique, la communication de Pierre Albert Castanet se fonde sur l'histoire contextuelle et interactive du «populaire» et du «savant». S'attachant notamment à l'instrumentarium symbolique des rockers (orgue électrique, guitare électrique, guitare basse, batterie), l'article se penche sur quelques études de cas allant des musiques de Pierre Henry à Philippe Manoury en passant par celles de Cathy Berberian, György Ligeti ou Tristan Murail...

\section{ABSTRACT}

For half a century, rock music has now been an ideological factor of our everyday mythology, and it has attracted several contemporary composers (John Adams, Luciano Berio, Steve Martland, Theo Loevendie, etc.). This essay examines the history of the contexts and the interactions of the "popular" and the "learned." By focusing on 
rockers' symbolic instrumentarium (electric organ, electric guitar, bass, drums), this essay studies an array of cases from Pierre Henry's to Philippe Manoury's music, as well as music from Cathy Berberan, György Ligeti and Tristan Murail. 